QUARTERLY OF APPLIED MATHEMATICS

VOLUME LXVIII, NUMBER 4

DECEMBER 2010, PAGES 671-699

S 0033-569X(2010)01183-5

Article electronically published on September 15, 2010

\title{
GLOBAL EXISTENCE AND STABILITY OF MILD SOLUTIONS TO THE INELASTIC BOLTZMANN SYSTEM FOR GAS MIXTURES
}

\author{
By \\ SEUNG-YEAL HA (Department of Mathematical Sciences, Seoul National University, Seoul \\ 151-747, Korea) \\ AND \\ SE EUN NOH (Department of Mathematical Sciences, Seoul National University, Seoul 151-747, \\ Korea) \\ Abstract. We study the global existence and uniform stability estimate of mild \\ solutions to the inelastic Boltzmann system for gas mixtures, when initial data are small \\ and decay exponentially in phase space, and we also provide a general multi-dimensional \\ Bony-type potential which yields a priori weighted two-point correlation estimates in \\ phase space to the mild solutions with finite mass and energy without any smallness \\ restriction.
}

1. Introduction. The purpose of this paper is to establish the global existence of mild solutions to the inelastic Boltzmann system and study their qualitative dynamic behavior such as the asymptotic completeness and uniform stability with respect to initial data. Consider a dilute binary gas mixture consisting of two species $A$ and $B$ undergoing inelastic collisions in the collision process. We assume that particles are electronically neutral so that the Boltzmann equation (BE in short) can be effectively used for the description of dynamics of the gas mixture. Let $F_{A}$ and $F_{B}$ be the oneparticle distribution functions for species $A$ and $B$ respectively. Then $F_{A}$ and $F_{B}$ are

Received February 23, 2009.

2000 Mathematics Subject Classification. Primary 35A05, 35B65, 78A35.

Key words and phrases. Boltzmann system, gas mixture, Lyapunov functional, multi-dimensional Bony functional, nonlinear functional approach.

The work of S.-Y. Ha is partially supported by KRF-2008-C00023 and research grant of CNS-SNU, and the work of S. Noh is supported by the BK21-Mathematical Division of SNU.

E-mail address: syha@snu.ac.kr

WWW address: http://www. math.snu.ac.kr/〜syha

E-mail address: senoh@math.snu.ac.kr

(C)2010 Brown University

Reverts to public domain 28 years from publication 
governed by the Boltzmann system with inelastic collision operators [12]:

$$
\begin{aligned}
& \partial_{t} F_{A}+\xi \cdot \nabla_{x} F_{A}=Q^{A A}\left(F_{A}, F_{A}\right)+Q^{A B}\left(F_{A}, F_{B}\right), \quad x, \xi \in \mathbb{R}^{3}, t>0, \\
& \partial_{t} F_{B}+\xi \cdot \nabla_{x} F_{B}=Q^{B B}\left(F_{B}, F_{B}\right)+Q^{B A}\left(F_{B}, F_{A}\right), \\
& \left(F_{A}, F_{B}\right)(x, \xi, 0)=\left(F_{A 0}, F_{B 0}\right)(x, \xi),
\end{aligned}
$$

where $Q^{\alpha \beta}\left(F_{\alpha}, F_{\beta}\right)$ represents the binary collision operator modeling interactions between particles of two species $\alpha$ and $\beta$; its specific form will be addressed in the next section. For the mild formulation of the system (1.1), it is convenient to introduce auxiliary functions along the particle trajectory as in $[23]$ :

$$
F_{\alpha}^{\sharp}(x, \xi, t):=F_{\alpha}(x+t \xi, \xi, t), \quad Q^{\alpha \beta \sharp}\left(F_{\alpha}, F_{\beta}\right)(x, \xi, t):=Q^{\alpha \beta}\left(F_{\alpha}, F_{\beta}\right)(x+t \xi, \xi, t) .
$$

We integrate (1.1) along the particle trajectory to find mild forms:

$$
\begin{aligned}
& F_{A}^{\sharp}(x, \xi, t)=F_{A 0}(x, \xi)+\int_{0}^{t}\left[Q^{A A \sharp}\left(F_{A}, F_{A}\right)+Q^{A B \sharp}\left(F_{A}, F_{B}\right)\right](x, \xi, s) d s, \\
& F_{B}^{\sharp}(x, \xi, t)=F_{B 0}(x, \xi)+\int_{0}^{t}\left[Q^{B B \sharp}\left(F_{B}, F_{B}\right)+Q^{B A \sharp}\left(F_{B}, F_{A}\right)\right](x, \xi, s) d s .
\end{aligned}
$$

We recall the definitions of a mild solution and the asymptotic completeness of the system (1.1).

Definition 1.1. 1. For $T \in(0, \infty]$, let $F_{A}, F_{B} \in C\left([0, T) ; L_{+}^{1}\left(\mathbb{R}^{6}\right)\right)$ be the mild solutions of (1.1) with nonnegative initial data $f_{A 0}, f_{B 0}$ if and only if $F_{A}$ and $F_{B}$ satisfy the integral equations (1.2) in a pointwise sense for all $t \in[0, T)$ and a.e $(x, \xi) \in \mathbb{R}^{6}$.

2. The Boltzmann system (1.1) satisfies "asymptotic completeness" in the $L^{1}$-sense if and only if for every global solution $F_{\alpha} \in C\left([0, T) ; L_{+}^{1}\left(\mathbb{R}^{6}\right)\right), \alpha \in\{A, B\}$ to (1.1), there exists the time-asymptotic state $F_{\alpha+}$ satisfying

$$
\lim _{t \rightarrow \infty}\left\|S(t) F_{\alpha 0}-S_{0}(t) F_{\alpha+}\right\|_{L^{1}}=0
$$

where $S(t)$ and $S_{0}(t)$ denote solution operators for the full Boltzmann system (1.1) and the free transport equation (1.3) respectively.

$$
\partial_{t} F_{\alpha}+\xi \cdot \nabla_{x} F_{\alpha}=0 .
$$

We next briefly review the mathematical results on the spatially inhomogeneous $\mathrm{BE}$ near a vacuum regime. For the corresponding results near a global Maxwellian regime, we refer readers to the survey article by Villani [27. For a single component gas, the global existence theories of mild and classical solutions have been studied in [4, 22, 23, 24, and uniform stability of classical and mild solutions with respect to initial data has been addressed in [13, 17, 19]. On the other hand, for kinetic models for the granular flow, there has been much intensive research on the spatially homogeneous inelastic Boltzmann equation [6, 7, 8, 9, 11, 14, whereas there are few available results on the well-posedness issues to the spatially inhomogeneous Boltzmann equation, for example the existence of mild solutions [5], uniform $L^{1}$-stability [18, for the one-dimensional model (see [16] for numerical issues). Recently Alonso studied the global existence of mild solutions to the one-component inelastic gas system using the celebrated Kaniel-Shinbrot argument [23]. See 28 for the current state of the art in this field. 
In this paper, we extend Alonso's result [1] for one-component gas to the multicomponent case and provide several dynamical estimates on the asymptotic completeness, uniform $L^{1}$-stability and a priori two-point correlation estimates in phase-space. For multi-component gas mixture with elastic collisions, some kinetic models [15, 21] with simplified collision operators such as a linear BGK-type operator have been used, and then several issues on the hydrodynamic limit, asymptotic analysis on the evaporation, condensation problem, and gas separations have been studied in [2, 3, 25, 26, 29, 30.

The rest of this paper is organized as follows. In Section 2, we describe the main framework for existence theory, which corresponds to Alonso's setting for a single-component case, and summarize the main results. In Section 3, we provide global mild solutions using similar arguments as in [19, employing the Kaniel-Shinbrot's method in the set consisting of small and exponentially decaying functions. In Section 4, we introduce an approximate Boltzmann system and study several estimates for the mollified approximate solutions. In Section 5, we introduce a generalized collision potential and provide a time-decay estimate along the mild solutions constructed in Section 3. As a byproduct of the time-decay of the generalized collision potential, we provide a simple proof for the $L^{1}$-asymptotic completeness for the inelastic Boltzmann system (1.1). In Section 6, we study a uniform weighted $L^{1}$-stability estimate for the mild solutions with respect to initial data using the nonlinear functional. Finally, Section 7 is devoted to the construction of a generalized Bony potential and its time-decay estimates for the mild solutions with finite mass and energy. The novelty of this potential is that the nonlinearity due to collision operators cancels in the time-decay estimate and hence the time-decay estimate of this potential yields the uniform estimate for the two-point weighted correlations.

2. Discussion of assumptions and main results. In this section, we discuss the main assumptions and results on the well-posedness of the system (1.1).

2.1. Main assumptions. In this part, we describe the main framework employed in this paper. We first discuss an inelastic collision process that conserves the mass and momentum but dissipates the energy. Suppose two particles $\alpha$ and $\beta$ with masses $m_{\alpha}, m_{\beta}$ and velocities $\xi$ and $\xi_{*}$ are employed in the collision process, and $\bar{\xi}$ and $\bar{\xi}_{*}$ denote the post-collisional velocities resulting from pre-collisional velocities $\left(\xi, \xi_{*}\right)$ :

$$
\begin{aligned}
m_{\alpha} \bar{\xi}+m_{\beta} \bar{\xi}_{*} & =m_{\alpha} \xi+m_{\beta} \xi_{*}, \\
\left(\bar{\xi}-\bar{\xi}_{*}\right) \cdot \omega & =-e\left(\xi-\xi_{*}\right) \cdot \omega,
\end{aligned}
$$

where $\omega \in \mathbf{S}^{2}$ and $e=e\left(\xi, \xi_{*}, \omega\right) \in[0,1]$ represent the free parameter for the scattering angle and a restitution coefficient, respectively. The inelastic nature of the collision process is encoded in the restitution coefficient $e . e=0$ and 1 correspond to the elastic collision and sticky collision respectively. This relation (2.1) yields

$$
\begin{aligned}
\bar{\xi} & =\xi+\frac{m_{\beta}}{m_{\alpha}+m_{\beta}}(1+e)\left[\left(\xi_{*}-\xi\right) \cdot \omega\right] \omega, \\
\bar{\xi}_{*} & =\xi_{*}-\frac{m_{\alpha}}{m_{\alpha}+m_{\beta}}(1+e)\left[\left(\xi_{*}-\xi\right) \cdot \omega\right] \omega .
\end{aligned}
$$


Note that the relation (2.2) leads to the decay of kinetic energy:

$$
m_{\alpha}|\bar{\xi}|^{2}+m_{\beta}\left|\bar{\xi}_{*}\right|^{2}=m_{\alpha}|\xi|^{2}+m_{\beta}\left|\xi_{*}\right|^{2}-\frac{m_{\alpha} m_{\beta}}{m_{\alpha}+m_{\beta}}\left(1-e^{2}\right)\left[\left(\xi-\xi_{*}\right) \cdot \omega\right]^{2} .
$$

In the sequel, we denote by the pair $\left(\xi^{\prime}, \xi_{*}^{\prime}\right)$ the pre-collisional velocities resulting in $\left(\xi, \xi_{*}\right)$ after the inelastic collision process (2.1), i.e.,

$$
\begin{aligned}
\xi & =\xi^{\prime}+\frac{m_{\beta}}{m_{\alpha}+m_{\beta}}\left(1+e^{\prime}\right)\left[\left(\xi_{*}^{\prime}-\xi^{\prime}\right) \cdot \omega\right] \omega, \\
\xi_{*} & =\xi_{*}^{\prime}-\frac{m_{\alpha}}{m_{\alpha}+m_{\beta}}\left(1+e^{\prime}\right)\left[\left(\xi_{*}^{\prime}-\xi^{\prime}\right) \cdot \omega\right] \omega,
\end{aligned}
$$

where $e^{\prime}=e\left(\xi^{\prime}, \xi_{*}^{\prime}, \omega\right)$. Note that $\xi^{\prime}$ and $\xi_{*}^{\prime}$ are different from the $\bar{\xi}$ and $\bar{\xi}_{*}$ (for the elastic collision, they are just the same because the collision process is micro-reversible and measuring-preserving).

We next return to the functional setting. For this, we introduce a local maxwellian

$$
M_{\alpha}(x, \xi):=e^{-m_{\alpha}\left(p|x|^{2}+q|\xi|^{2}\right)}, \quad p, q>0, \alpha \in\{A, B\},
$$

and define a set and norm bounded by the local maxwellian:

$$
\begin{aligned}
& \mathcal{S}_{\alpha}:=\left\{F \in C\left(\mathbb{R}_{+} ; L^{1}\left(\mathbb{R}^{3} \times \mathbb{R}^{3}\right)\right)|| F^{\sharp}(x, \xi, t) \mid \leq \delta_{\alpha} M_{\alpha}(x, \xi) \text { for some } \delta_{\alpha}>0\right\}, \\
& \mathcal{S}_{\alpha, T}:=\left\{F \in C^{0}\left([0, T] \times \mathbb{R}^{3} \times \mathbb{R}^{3}\right) \mid\|F\|_{\alpha} \leq 2 \delta_{\alpha}, \text { for some } \delta_{\alpha}\right\} \quad \text { for some T, } \\
& \left\|F^{\sharp}\right\|_{\alpha}:=\sup _{0 \leq t<\infty} \sup _{x, \xi \in \mathbb{R}^{3}}\left[e^{m_{\alpha}\left(p|x|^{2}+q|\xi|^{2}\right)}\left|F^{\sharp}(x, \xi, t)\right|\right], \\
& \left\|F_{\alpha}(t)\right\|_{L_{\alpha, r}^{1}}:=\left\|F_{\alpha}(\cdot, \cdot, t)\right\|_{L^{1}\left(\varphi_{\alpha, r} d x d \xi\right)}, \quad \varphi_{\alpha, r}(\xi):=\left(1+m_{\alpha}|\xi|^{2}\right)^{\frac{r}{2}} .
\end{aligned}
$$

Below, we list assumptions imposed on the restitution coefficient and initial data:

- $(\mathcal{C} 1): e$ is the function of impact velocity, i.e.

$$
e=e\left(\left|\left(\xi-\xi_{*}\right) \cdot \omega\right|\right) \text {. }
$$

- $(\mathcal{C} 2): z \mapsto e(z)$ is an absolutely continuous function from $[0, \infty)$ to $(0,1]$.

- $(\mathcal{C} 3): z \mapsto z e(z)$ is strictly increasing.

- $(\mathcal{C} 4)$ : Integrability condition:

$$
A_{\alpha \beta}(x):=\int_{0}^{\frac{\pi}{2}} \frac{\exp \left\{-\frac{m_{\alpha} m_{\beta}}{m_{\alpha}+m_{\beta}}\left(1-e(x \cos \theta)^{2}\right)(x \cos \theta)^{2}\right\}}{e(x \cos \theta)(e(x \cos \theta)+x \cos \theta \dot{e}(x \cos \theta))} \sin \theta d \theta \in L^{\infty}([0, \infty)) \text {. }
$$

- $(\mathcal{C} 5)$ : Initial data satisfy the regularity assumption and are sufficiently small:

$$
F_{\alpha 0} \in C\left(\mathbb{R}^{3} \times \mathbb{R}^{3}\right) \cap \mathcal{S}_{\alpha}, \quad 0<\delta_{\alpha} \ll 1, \quad \alpha \in\{A, B\} .
$$

REMARK 2.1. The conditions on the restitution coefficient $e$ were adopted from [1 for a single component inelastic gas system. We add some comments on the assumptions on $e$ as follows.

(i) The condition $(\mathcal{C} 2)$ guarantees

$$
J=\left|\frac{\partial\left(\bar{\xi}, \bar{\xi}_{*}\right)}{\partial\left(\xi, \xi_{*}\right)}\right|=e\left(\left|\left(\xi-\xi_{*}\right) \cdot \omega\right|\right)+\left|\left(\xi-\xi_{*}\right) \cdot \omega\right| \frac{d e}{d z}\left(\left|\left(\xi-\xi_{*}\right) \cdot \omega\right|\right), \quad \text { a.e. }
$$

(ii) The condition $(\mathcal{C} 3)$ implies the positivity of $J\left(\left|\left(\xi-\xi_{*}\right) \cdot \omega\right|\right)$ so that the collision transformation (2.2) is reversible, but not measure-preserving. 
(iii) The integrability condition $(\mathcal{C} 4)$ is needed for a technical reason. However, many cases of restitution coefficients appearing in the context of the viscoelastic and elastic cases satisfy this condition (for detailed explanation see [1]).

2.2. Main results. In this part, we briefly summarize the main results that will follow after this section. Our first main result is the well-posedness of the Boltzmann system (1.1) with assumptions $(\mathcal{C} 1)-(\mathcal{C} 5)$. The global existence of mild solutions in the set $\mathcal{S}_{A} \times \mathcal{S}_{B}$ follows from the standard Kaniel-Shinbrot arguments as in 1, 19, 23. On the other hand, the asymptotic completeness and uniform $L^{1}$-stability will be studied via several collision potentials, which basically measure future collisions between particles. The first main result is described in the following theorem.

Theorem 2.1. Suppose that the main assumptions $(\mathcal{C} 1)-(\mathcal{C} 5)$ hold. Then there exist the unique mild solution $F_{A}, F_{B} \in C\left(\mathbb{R}_{+} ; L^{1}\left(\mathbb{R}^{6}\right)\right)$ to the Boltzmann system (1.1) and their time-asymptotic limits $F_{A+}, F_{B+}$ satisfying the uniform boundedness and the uniform $L_{\alpha, r}^{1}$-stability estimate:

$$
\begin{aligned}
& \text { (i) } 0 \leq F_{\alpha}^{\sharp} \leq 2 \delta_{\alpha} M_{\alpha}, \quad \alpha \in\{A, B\} . \\
& \text { (ii) } \lim _{t \rightarrow \infty} \sum_{\alpha \in\{A, B\}} \| F_{\alpha}^{\sharp}(t)-F_{\alpha+\|_{L_{\alpha, r}^{1}}}=0 . \\
& \text { (iii) } \sup _{t \geq 0} \sum_{\alpha \in\{A, B\}}\left\|F_{\alpha}(t)-\bar{F}_{\alpha}(t)\right\|_{L_{\alpha, r}^{1}} \leq G \sum_{\alpha \in\{A, B\}}\left\|F_{\alpha 0}-\bar{F}_{\alpha 0}\right\|_{L_{\alpha, r}^{1},},
\end{aligned}
$$

where $G$ is a positive constant independent of $t$.

REMARK 2.2. For $\varphi_{\alpha, 0}:=1$, we recover the well-known uniform $L^{1}$-stability estimate (see [13, 17, 19]).

The second main result is the weighted two-point correlation estimate in one-particle phase-space $\mathbb{R}^{6}$ for mild solutions with finite mass and energy. We will construct a robust Bony-type collision potential and as a byproduct of time-decay of this potential, we obtain a uniform estimate for the time-phase space integral of the two-point correlation function. Let $\omega: \mathbb{R}^{3} \rightarrow \mathbb{R}$ be a $C^{2}$-convex, locally integrable and radially symmetric function, i.e.,

$$
\omega(x)=\varphi(|x|), \quad \varphi^{\prime} \leq 0, \quad \varphi^{\prime} \in L^{\infty}(\mathbb{R}) .
$$

ThEOREM 2.2. Let $F=\left(F_{A}, F_{B}\right)$ be the mild solutions to the system (1.1) with finite mass and energy. Then we have the following estimate:

$$
\begin{gathered}
\int_{0}^{\infty} \int_{\mathbb{R}^{12}}\left(\xi_{*}-\xi\right)^{T} H\left(\xi_{*}-\xi\right)\left(m_{A} F_{A}+m_{B} F_{B}\right)(x, \xi, t)\left(m_{A} F_{A}+m_{B} F_{B}\right)\left(y, \xi_{*}, t\right) d \xi_{*} d \xi d y d x d t \\
<C\left(F_{0}\right)
\end{gathered}
$$

where we used a handy notation $H:=\left[\partial_{i} \partial_{j} \omega\right], \partial_{i}:=\frac{\partial}{\partial x_{i}}$ for the Hessian matrix of a $C^{2}$-convex function $\omega$, and $C\left(F_{0}\right)$ is a positive constant depending only on the mass and energy of the initial data $F_{0}=\left(F_{A 0}, F_{B 0}\right)$.

REMARK 2.3. 1. Note that

$$
\left(\xi_{*}-\xi\right)^{T} H\left(\xi_{*}-\xi\right)>0 .
$$


2. For an a priori estimate of Theorem 2.2, we only need initial data with finite mass and energy. The smallness of the initial data is not used in the analysis.

3. In (2.5), the condition $\varphi^{\prime} \in L^{\infty}(\mathbb{R})$ can be dropped using the exponential decay of $F_{A}$ and $F_{B}$ in the $x$-variable.

3. Global existence of mild solutions. In this section, we present basic properties of the collision operator and briefly discuss the global existence of mild solutions employing the modified Kaniel-Shinbrot scheme.

3.1. Basic estimates. In this part, we review basic properties of the collision operators and present some basic estimates for the global existence of mild solutions. We now introduce the explicit form of collision operators between two species $\alpha$ and $\beta$ :

$$
\begin{aligned}
Q^{\alpha \beta}\left(F_{\alpha}, F_{\beta}\right)(x, \xi, t) & :=Q_{+}^{\alpha \beta}\left(F_{\alpha}, F_{\beta}\right)(x, \xi, t)-Q_{-}^{\alpha \beta}\left(F_{\alpha}, F_{\beta}\right)(x, \xi, t), \\
Q_{+}^{\alpha \beta}\left(F_{\alpha}, F_{\beta}\right)(x, \xi, t) & =\int_{\mathbb{R}^{3} \times \mathbf{S}^{2}} \frac{\left|\left(\xi-\xi_{*}\right) \cdot \omega\right|}{e^{\prime} J^{\prime}} F_{\alpha}\left(\xi^{\prime}\right) F_{\beta}\left(\xi_{*}^{\prime}\right) d \omega d \xi_{*}, \\
Q_{-}^{\alpha \beta}\left(F_{\alpha}, F_{\beta}\right)(x, \xi, t) & =\int_{\mathbb{R}^{3} \times \mathbf{S}^{2}}\left|\left(\xi-\xi_{*}\right) \cdot \omega\right| F_{\alpha}(\xi) F_{\beta}\left(\xi_{*}\right) d \omega d \xi_{*} \\
& =F_{\alpha}(x, \xi, t) \underbrace{\int_{\mathbb{R}^{3} \times \mathbf{S}^{2}}\left|\left(\xi-\xi_{*}\right) \cdot \omega\right| F_{\beta}\left(\xi_{*}\right) d \omega d \xi_{*}}_{R^{\alpha \beta}\left(F_{\beta}\right)(x, \xi, t)} .
\end{aligned}
$$

Here $\left(\xi^{\prime}, \xi_{*}^{\prime}\right)$ are the pre-collision velocities resulting in $\left(\xi, \xi_{*}\right)$ (see the relation (2.2)), $Q_{+}^{\alpha \beta}\left(F_{\alpha}, F_{\beta}\right)$ and $Q_{-}^{\alpha \beta}\left(F_{\alpha}, F_{\beta}\right)$ are known as the gain and loss operator, respectively, and we use the abbreviated notation $F_{\alpha}(\xi)=F_{\alpha}(x, \xi, t)$. In an inelastic collision process, particles are commonly modeled as hard spheres. This is why the kernel $\left|\left(\xi-\xi_{*}\right) \cdot \omega\right|$ is used for the collision operator.

Lemma 3.1. Let $F_{\alpha}$ and $F_{\beta}$ be measurable functions decaying at infinity in phase space. Then for any measurable function $\psi=\psi(\xi)$, we have

(i) $\int_{\mathbb{R}^{3}} \psi(\xi) Q^{\alpha \beta}\left(F_{\alpha}, F_{\beta}\right)(\xi) d \xi$

$$
=\int_{\mathbb{R}^{6} \times \mathbf{S}^{2}}\left|\left(\xi-\xi_{*}\right) \cdot \omega\right| F_{\alpha}(\xi) F_{\beta}\left(\xi_{*}\right)[\psi(\bar{\xi})-\psi(\xi)] d \omega d \xi_{*} d \xi .
$$

(ii) $\int_{\mathbb{R}^{3}} \psi(\xi) Q^{\alpha \alpha}\left(F_{\alpha}, F_{\alpha}\right)(\xi) d \xi$

$$
=\frac{1}{2} \int_{\mathbb{R}^{6} \times \mathbf{S}^{2}}\left|\left(\xi-\xi_{*}\right) \cdot \omega\right| F_{\alpha}(\xi) F_{\alpha}\left(\xi_{*}\right)\left[\psi(\bar{\xi})+\psi\left(\bar{\xi}_{*}\right)-\psi(\xi)-\psi\left(\xi_{*}\right)\right] d \omega d \xi_{*} d \xi .
$$

Proof. (i) Note that $\left(\xi-\xi_{*}\right) \cdot \omega=e^{\prime}\left(\xi^{\prime}-\xi_{*}^{\prime}\right) \cdot \omega$ and then the change of variables $\left(\xi, \xi_{*}\right) \leftrightarrow\left(\xi^{\prime}, \xi_{*}^{\prime}\right)$ yield

$$
\begin{aligned}
\int_{\mathbb{R}^{3}} \psi & (\xi) \\
= & Q_{+}^{\alpha \beta}\left(F_{\alpha}, F_{\beta}\right)(\xi) d \xi \\
= & \frac{\left|\left(\xi-\xi_{*}\right) \cdot \omega\right|}{e^{\prime} J^{\prime}} F_{\alpha}\left(\xi^{\prime}\right) F_{\beta}\left(\xi_{*}^{\prime}\right) \psi(\xi) d \omega d \xi_{*} d \xi \\
& \int_{\mathbb{R}^{6} \times \mathbf{S}^{2}}\left|\left(\xi-\xi_{*}\right) \cdot \omega\right| F_{\alpha}(\xi) F_{\beta}\left(\xi_{*}\right) \psi(\bar{\xi}) d \omega d \xi_{*} d \xi
\end{aligned}
$$


where $\bar{\xi}$ represents the post-collisional velocity for $\xi$.

(ii) We use the fact that

$$
\left|\left(\xi-\xi_{*}\right) \cdot \omega\right|=\left|\left(\xi_{*}-\xi\right) \cdot \omega\right|
$$

and the change of variables $\left(\xi, \xi_{*}\right) \leftrightarrow\left(\xi_{*}, \xi\right)$ to see that

$$
\begin{aligned}
\int_{\mathbb{R}^{3}} \psi(\xi) & Q^{\alpha \alpha}\left(F_{\alpha}, F_{\alpha}\right)(\xi) d \xi \\
= & \int_{\mathbb{R}^{6} \times \mathbf{S}^{2}}\left|\left(\xi-\xi_{*}\right) \cdot \omega\right|\left(\frac{F_{\alpha}\left(\xi^{\prime}\right) F_{\alpha}\left(\xi_{*}^{\prime}\right)}{e^{\prime} J^{\prime}}-F_{\alpha}(\xi) F_{\alpha}\left(\xi_{*}\right)\right) \psi(\xi) d \omega d \xi_{*} d \xi \\
= & \int_{\mathbb{R}^{6} \times \mathbf{S}^{2}}\left|\left(\xi-\xi_{*}\right) \cdot \omega\right|\left(\frac{F_{\alpha}\left(\xi^{\prime}\right) F_{\alpha}\left(\xi_{*}^{\prime}\right)}{e^{\prime} J^{\prime}}-F_{\alpha}(\xi) F_{\alpha}\left(\xi_{*}\right)\right) \psi\left(\xi_{*}\right) d \omega d \xi_{*} d \xi
\end{aligned}
$$

We add two equalities and apply (i) to complete the proof.

Equipped with the above lemma, we can show that the collision operators satisfy the following collisional invariant properties.

Proposition 3.1. Let $F_{\alpha}$ and $F_{\beta}$ be measurable functions decaying sufficiently fast at infinity in phase space. Then we have

$$
\begin{aligned}
& \text { (i) } \int_{\mathbb{R}^{3}} Q^{\alpha \alpha}\left(F_{\alpha}, F_{\alpha}\right) d \xi=\int_{\mathbb{R}^{3}} Q^{\alpha \beta}\left(F_{\alpha}, F_{\beta}\right) d \xi=0 . \\
& \text { (ii) } \int_{\mathbb{R}^{3}} \xi Q^{\alpha \alpha}\left(F_{\alpha}, F_{\alpha}\right) d \xi=0 . \\
& \text { (iii) } \int_{\mathbb{R}^{3}} m_{\alpha} \xi Q^{\alpha \beta}\left(F_{\alpha}, F_{\beta}\right) d \xi+\int_{\mathbb{R}^{3}} m_{\beta} \xi Q^{\beta \alpha}\left(F_{\beta}, F_{\alpha}\right) d \xi=0 . \\
& \text { (iv) } \int_{\mathbb{R}^{3}} m_{\alpha}|\xi|^{2} Q^{\alpha \beta}\left(F_{\alpha}, F_{\beta}\right) d \xi+\int_{\mathbb{R}^{3}} m_{\beta}|\xi|^{2} Q^{\beta \alpha}\left(F_{\beta}, F_{\alpha}\right) d \xi \leq 0 .
\end{aligned}
$$

Proof. The proof is the direct consequence of Lemma 3.1 and (2.3).

We now present two basic estimates.

Lemma 3.2. For $\alpha, \beta \in\{A, B\}$, we have

$$
\begin{aligned}
& M_{\alpha}^{-1}(x, \xi) \int_{0}^{\infty} M_{\alpha}\left(x+t\left(\xi-\xi^{\prime}\right), \xi^{\prime}\right) M_{\beta}\left(x+t\left(\xi-\xi_{*}^{\prime}\right), \xi_{*}^{\prime}\right) d t \\
& \quad \leq\left(\frac{\pi}{p m_{\beta}}\right)^{\frac{1}{2}}\left|\xi-\xi_{*}\right|^{-1} e^{-q m_{\beta}\left|\xi_{*}\right|^{2}} e^{-\frac{m_{\alpha} m_{\beta}}{m_{\alpha}+m_{\beta}} q\left(1-e^{\prime 2}\right)\left(\left(\xi^{\prime}-\xi_{*}^{\prime}\right) \cdot \omega\right)^{2}} .
\end{aligned}
$$

Proof. Note that momentum conservation (2.1) and (2.4) yield

$$
\begin{aligned}
m_{\alpha}\left|x+t\left(\xi-\xi^{\prime}\right)\right|^{2}+m_{\beta}\left|x+t\left(\xi-\xi_{*}^{\prime}\right)\right|^{2} \\
\quad=\left(m_{\alpha}+m_{\beta}\right)|x+t \xi|^{2}-2 t(x+t \xi) \cdot\left(m_{\alpha} \xi^{\prime}+m_{\beta} \xi_{*}^{\prime}\right)+t^{2}\left(m_{\alpha}\left|\xi^{\prime}\right|^{2}+m_{\beta}\left|\xi_{*}^{\prime}\right|^{2}\right) \\
\quad=\left(m_{\alpha}+m_{\beta}\right)|x+t \xi|^{2}-2 t(x+t \xi) \cdot\left(m_{\alpha} \xi+m_{\beta} \xi_{*}\right)+t^{2}\left(m_{\alpha}|\xi|^{2}+m_{\beta}\left|\xi_{*}\right|^{2}\right) \\
\quad+t^{2} \frac{m_{\alpha} m_{\beta}}{m_{\alpha}+m_{\beta}}\left(1-e^{\prime 2}\right)\left(\left(\xi^{\prime}-\xi_{*}^{\prime}\right) \cdot \omega\right)^{2} \\
\quad=m_{\alpha}|(x+t \xi)-t \xi|^{2}+m_{\beta}\left|(x+t \xi)-t \xi_{*}\right|^{2}+t^{2} \frac{m_{\alpha} m_{\beta}}{m_{\alpha}+m_{\beta}}\left(1-e^{\prime 2}\right)\left(\left(\xi^{\prime}-\xi_{*}^{\prime}\right) \cdot \omega\right)^{2} \\
\quad=m_{\alpha}|x|^{2}+m_{\beta}\left|x+t\left(\xi-\xi_{*}\right)\right|^{2}+t^{2} \frac{m_{\alpha} m_{\beta}}{m_{\alpha}+m_{\beta}}\left(1-e^{\prime 2}\right)\left(\left(\xi^{\prime}-\xi_{*}^{\prime}\right) \cdot \omega\right)^{2} .
\end{aligned}
$$


We use the above relation to see that

$$
\begin{aligned}
\int_{0}^{\infty} M_{\alpha}\left(x+t\left(\xi-\xi^{\prime}\right), \xi^{\prime}\right) M_{\beta}\left(x+t\left(\xi-\xi_{*}^{\prime}\right), \xi_{*}^{\prime}\right) d t \\
\quad=\int_{0}^{\infty} M_{\alpha}(x, \xi) M_{\beta}\left(x+t\left(\xi-\xi_{*}\right), \xi_{*}\right) e^{-\left(p t^{2}+q\right) \frac{m_{\alpha} m_{\beta}}{m_{\alpha}+m_{\beta}}\left(1-e^{\prime 2}\right)\left(\left(\xi^{\prime}-\xi_{*}^{\prime}\right) \cdot \omega\right)^{2}} d t \\
\quad \leq M_{\alpha}(x, \xi) e^{-q m_{\beta}\left|\xi_{*}\right|^{2}-q \frac{m_{\alpha} m_{\beta}}{m_{\alpha}+m_{\beta}}\left(1-e^{\prime 2}\right)\left(\left(\xi^{\prime}-\xi_{*}^{\prime}\right) \cdot \omega\right)^{2}} \int_{0}^{\infty} e^{-p m_{\beta}\left|x+t\left(\xi-\xi_{*}\right)\right|^{2}} d t .
\end{aligned}
$$

On the other hand, we have

$$
\begin{aligned}
\int_{0}^{\infty} e^{-p m_{\beta}\left|x+t\left(\xi-\xi_{*}\right)\right|^{2}} d t & \leq \int_{-\infty}^{\infty} e^{-p m_{\beta}\left|\xi-\xi_{*}\right|^{2}\left(\frac{x \cdot\left(\xi-\xi_{*}\right)}{\left|\xi-\xi_{*}\right|^{2}}+t\right)^{2}} d t \\
& \leq \int_{-\infty}^{\infty} e^{-p m_{\beta}\left|\xi-\xi_{*}\right|^{2} t^{2}} d t=\sqrt{\frac{\pi}{p m_{\beta}}} \frac{1}{\left|\xi-\xi_{*}\right|},
\end{aligned}
$$

where we used

$$
\begin{aligned}
\left|x+t\left(\xi-\xi_{*}\right)\right|^{2} & =|x|^{2}-\frac{\left(x \cdot\left(\xi-\xi_{*}\right)\right)^{2}}{\left|\xi-\xi_{*}\right|^{2}+\left|\xi-\xi_{*}\right|^{2}\left(\frac{x \cdot\left(\xi-\xi_{*}\right)}{\left|\xi-\xi_{*}\right|^{2}}+t\right)^{2}} \\
& \geq\left|\xi-\xi_{*}\right|^{2}\left(\frac{x \cdot\left(\xi-\xi_{*}\right)}{\left|\xi-\xi_{*}\right|^{2}}+t\right)^{2} .
\end{aligned}
$$

We combine (3.2) and (3.3) to get the desired result.

Lemma 3.3. Let $(f, g) \in \mathcal{S}_{\alpha} \times \mathcal{S}_{\beta}$. Then we have following estimate.

$$
\int_{0}^{t}\left|Q_{+}^{\alpha \beta \sharp}(f, g)(x, \xi, \tau)\right| d \tau \leq C_{\alpha \beta}\|f\|_{\alpha}\|g\|_{\beta} M_{\alpha}(x, \xi),
$$

where $C_{\alpha \beta}$ is a positive constant:

$$
C_{\alpha \beta}:=2 \pi\left(\frac{\pi}{p m_{\beta}}\right)^{\frac{1}{2}}\left(\frac{\pi}{q m_{\beta}}\right)^{\frac{3}{2}}\left\|A_{\alpha \beta}\right\|_{\infty},
$$

where $A_{\alpha \beta}$ is defined in the assumption $(\mathcal{C} 4)$ in Section 2.

Proof. We use Lemma 3.1 and the Fubini theorem to see that

$$
\begin{aligned}
\int_{0}^{t} \mid & Q_{+}^{\alpha \beta \sharp}(f, g)(x, \xi, \tau) \mid d \tau \\
& =\int_{0}^{t}\left|\int_{\mathbb{R}^{3} \times \mathbf{S}^{2}} \frac{\left|\left(\xi-\xi_{*}\right) \cdot \omega\right|}{e^{\prime} J^{\prime}} f^{\sharp}\left(x+\tau\left(\xi-\xi^{\prime}\right), \xi^{\prime}, t\right) g^{\sharp}\left(x+\tau\left(\xi-\xi_{*}^{\prime}\right), \xi_{*}^{\prime}, t\right) d \omega d \xi_{*}\right| d \tau \\
& \leq\|f\|_{\alpha}\|g\|_{\beta} \int_{0}^{t} \int_{\mathbb{R}^{3} \times \mathbf{S}^{2}} \frac{\left|\left(\xi-\xi_{*}\right) \cdot \omega\right|}{e^{\prime} J^{\prime}} M_{\alpha}\left(x+\tau\left(\xi-\xi^{\prime}\right), \xi^{\prime}\right) M_{\beta}\left(x+\tau\left(\xi-\xi_{*}^{\prime}\right), \xi_{*}^{\prime}\right) d \omega d \xi_{*} d \tau \\
& \leq \sqrt{\frac{\pi}{p m_{\beta}}}\|f\|_{\alpha}\|g\|_{\beta} M_{\alpha}(x, \xi) \int_{\mathbb{R}^{3}} e^{-q m_{\beta}\left|\xi_{*}\right|^{2}}\left[\int_{\mathbf{S}^{2}} \frac{1}{e^{\prime} J^{\prime}} e^{-q \frac{m_{\alpha} m_{\beta}}{m_{\alpha}+m_{\beta}}\left(1-e^{\prime 2}\right)\left(\left(\xi^{\prime}-\xi_{*}^{\prime}\right) \cdot \omega\right)^{2}} d \omega\right] d \xi_{*} .
\end{aligned}
$$

Here we use the assumption $(\mathcal{C} 4)$ to get

$$
\int_{\mathbf{S}^{2}} \frac{1}{e^{\prime} J^{\prime}} e^{-q \frac{m_{\alpha} m_{\beta}}{m_{\alpha}+m_{\beta}}\left(1-e^{\prime 2}\right)\left(\left(\xi^{\prime}-\xi_{*}^{\prime}\right) \cdot \omega\right)^{2}} d \omega=2 \pi A_{\alpha \beta}\left(\left|\xi^{\prime}-\xi_{*}^{\prime}\right|\right),
$$

which proves this lemma. 
3.2. Global existence of mild solutions. In this part, we present a Kaniel-Shinbrot 23] scheme for (1.1). We use (3.1) to rewrite (1.1) as

$$
\begin{aligned}
& \partial_{t} F_{A}+\xi \cdot \nabla_{x} F_{A}+F_{A}\left[R^{A A}\left(F_{A}\right)+R^{A B}\left(F_{B}\right)\right]=Q_{+}^{A A}\left(F_{A}, F_{A}\right)+Q_{+}^{A B}\left(F_{A}, F_{B}\right), \\
& \partial_{t} F_{B}+\xi \cdot \nabla_{x} F_{B}+F_{B}\left[R^{B B}\left(F_{B}\right)+R^{B A}\left(F_{A}\right)\right]=Q_{+}^{B B}\left(F_{B}, F_{B}\right)+Q_{+}^{B A}\left(F_{B}, F_{A}\right),
\end{aligned}
$$

and define four sequences $\left\{u_{A}^{k}\right\},\left\{l_{A}^{k}\right\},\left\{u_{A}^{k}\right\},\left\{l_{A}^{k}\right\}, k \geq 1$ as the solutions of the following linear system:

$$
\begin{aligned}
& \frac{d}{d t} l_{A}^{(k+1) \sharp}+l_{A}^{(k+1) \sharp}\left[R^{A A \sharp}\left(u_{A}^{k}\right)+R^{A B \sharp}\left(u_{B}^{k}\right)\right]=Q_{+}^{A A \sharp}\left(l_{A}^{k}, l_{A}^{k}\right)+Q_{+}^{A B \sharp}\left(l_{A}^{k}, l_{B}^{k}\right), \\
& \frac{d}{d t} u_{A}^{(k+1) \sharp}+u_{A}^{(k+1) \sharp}\left[R^{A A \sharp}\left(l_{A}^{k}\right)+R^{A B \sharp}\left(l_{B}^{k}\right)\right]=Q_{+}^{A A \sharp}\left(u_{A}^{k}, u_{A}^{k}\right)+Q_{+}^{A B \sharp}\left(u_{A}^{k}, u_{B}^{k}\right), \\
& \frac{d}{d t} l_{B}^{(k+1) \sharp}+l_{B}^{(k+1) \sharp}\left[R^{B B \sharp}\left(u_{B}^{k}\right)+R^{B A \sharp}\left(u_{A}^{k}\right)\right]=Q_{+}^{B B \sharp}\left(l_{B}^{k}, l_{B}^{k}\right)+Q_{+}^{B A \sharp}\left(l_{B}^{k}, l_{A}^{k}\right), \\
& \frac{d}{d t} u_{B}^{(k+1) \sharp}+u_{B}^{(k+1) \sharp}\left[R^{A A \sharp}\left(l_{B}^{k}\right)+R^{B A \sharp}\left(l_{A}^{k}\right)\right]=Q_{+}^{B B \sharp}\left(u_{B}^{k}, u_{B}^{k}\right)+Q_{+}^{B A \sharp}\left(u_{B}^{k}, u_{A}^{k}\right), \\
& u_{A}^{k+1}(0)=F_{A 0}, \quad l_{A}^{k+1}(0)=F_{A 0}, \quad u_{B}^{k+1}(0)=F_{B 0}, \quad l_{B}^{k+1}(0)=F_{B 0} .
\end{aligned}
$$

Proposition 3.2. Let $T$ be a given positive number and $\alpha \in\{A, B\}$. With the initial data $F_{\alpha 0} \in \mathcal{S}_{\alpha}$, we assume that the iteration begins with the sufficiently small pairs $\left(l_{\alpha}^{0}(t), u_{\alpha}^{0}(t)\right)$ with $u_{\alpha}^{0}(t) \in \mathcal{S}_{\alpha, T}$ satisfying the beginning condition [23], i.e.,

$$
0 \leq l_{\alpha}^{0}(t) \leq l_{\alpha}^{1}(t) \leq u_{\alpha}^{1}(t) \leq u_{\alpha}^{0}(t)
$$

Then the linear system (3.5) has the unique solutions

$$
l_{\alpha}^{k \sharp}, u_{\alpha}^{k \sharp} \in \mathcal{S}_{\alpha, T} \quad \text { for all } k \geq 1
$$

satisfying

$$
0 \leq l_{\alpha}^{k-1}(t) \leq l_{\alpha}^{k}(t) \leq u_{\alpha}^{k}(t) \leq u_{\alpha}^{k-1}(t), \quad 0 \leq t<T
$$

Consequently, the sequences $\left\{l_{\alpha}^{k}\right\}$ and $\left\{u_{\alpha}^{k}\right\}$ converge in $\mathcal{S}_{\alpha, T}$.

Proof. In order to use mathematical induction we suppose that

$$
u_{\alpha}^{k} \leq u_{\alpha}^{k-1} \text { and } l_{\alpha}^{k-1} \leq l_{\alpha}^{k} \text {, equivalently } u_{\alpha}^{k \sharp} \leq u_{\alpha}^{(k-1) \sharp} \text { and } l_{\alpha}^{(k-1) \sharp} \leq l_{\alpha}^{k \sharp} \text {. }
$$

Then it is easy to see that for $\alpha, \beta \in\{A, B\}$ and $\alpha \neq \beta$

$$
\begin{aligned}
\int_{0}^{t}\left[R^{\alpha \alpha \sharp}\left(u_{\alpha}^{k}\right)+R^{\alpha \beta \sharp}\left(u_{\beta}^{k}\right)\right] d \tau & \leq \int_{0}^{t}\left[R^{\alpha \alpha \sharp}\left(u_{\alpha}^{k-1}\right)+R^{\alpha \beta \sharp}\left(u_{\beta}^{k-1}\right)\right] d \tau, \\
Q_{+}^{\alpha \alpha \sharp}\left(l_{\alpha}^{k}, l_{\alpha}^{k}\right)+Q_{+}^{\alpha \beta \sharp}\left(l_{\alpha}^{k}, l_{\beta}^{k}\right) & \geq Q_{+}^{\alpha \alpha \sharp}\left(l_{\alpha}^{k-1}, l_{\alpha}^{k-1}\right)+Q_{+}^{\alpha \beta \sharp}\left(l_{\alpha}^{k-1}, l_{\beta}^{k-1}\right) .
\end{aligned}
$$


We now integrate (3.5) along the particle trajectory $(x+s \xi, \xi, s), 0 \leq s \leq T$ to get

$$
\begin{aligned}
l_{\alpha}^{k \sharp}= & F_{\alpha 0} e^{-\int_{0}^{t}\left[R^{\alpha \alpha \sharp}\left(u_{\alpha}^{k-1}\right)+R^{\alpha \beta \sharp}\left(u_{\beta}^{k-1}\right)\right] d \tau} \\
+ & \int_{0}^{t} e^{-\int_{\tau}^{t}\left[R^{\alpha \alpha \sharp}\left(u_{\alpha}^{k-1}\right)+R^{\alpha \beta \sharp}\left(u_{\beta}^{k-1}\right)\right] d s}\left[Q_{+}^{\alpha \sharp}\left(l_{\alpha}^{k-1}, l_{\alpha}^{k-1}\right)+Q_{+}^{\alpha \beta \sharp}\left(l_{\alpha}^{k-1}, l_{\beta}^{k-1}\right)\right] \\
& \times d \tau, \\
l_{\alpha}^{k+1 \sharp}= & F_{\alpha 0} e^{-\int_{0}^{t}\left[R^{\alpha \alpha \sharp}\left(u_{\alpha}^{k}\right)+R^{\alpha \beta \sharp}\left(u_{\beta}^{k}\right)\right] d \tau} \\
+ & \int_{0}^{t} e^{-\int_{\tau}^{t}\left[R^{\alpha \alpha \sharp}\left(u_{\alpha}^{k}\right)+R^{\alpha \beta \sharp}\left(u_{\beta}^{k}\right)\right] d s}\left[Q_{+}^{\alpha \alpha \sharp}\left(l_{\alpha}^{k}, l_{\alpha}^{k}\right)+Q_{+}^{\alpha \beta \sharp}\left(l_{\alpha}^{k}, l_{\beta}^{k}\right)\right] d \tau,
\end{aligned}
$$

which proves the monotone increasing property of the sequence $\left\{l_{\alpha}^{k}\right\}$. We use Lemma 3.3 to see that

$$
\begin{aligned}
l_{\alpha}^{k+1 \sharp} & =F_{\alpha 0}+\int_{0}^{t}\left[Q_{+}^{\alpha \alpha \sharp}\left(l_{\alpha}^{k}, l_{\alpha}^{k}\right)+Q_{+}^{\alpha \beta \sharp}\left(l_{\alpha}^{k}, l_{\beta}^{k}\right)\right] d \tau \\
& \leq\left\|F_{\alpha 0}\right\|_{\alpha}+\left[C_{\alpha \alpha}\left\|l_{\alpha}^{k}\right\|_{\alpha}\left\|l_{\alpha}^{k}\right\|_{\alpha}+C_{\alpha \beta}\left\|l_{\alpha}^{k}\right\|_{\alpha}\left\|l_{\beta}^{k}\right\|_{\beta}\right] M_{\alpha}(x, \xi) \in \mathcal{S}_{\alpha, T} .
\end{aligned}
$$

The proof of $\left\{u_{\alpha}^{k}\right\}$ is similar and is omitted. Finally, each sequence $l_{A}^{k}, u_{A}^{k}, l_{B}^{k}$, and $u_{B}^{k}$ converges to $l_{A}, u_{A}, l_{B}$, and $u_{B}$ respectively, due to the boundedness and monotonicity of the sequence.

The next proposition says that the limits of iterative sequences for species $A$ and $B$ not only exist but also coincide with each other.

Proposition 3.3. Let $T$ be a given positive number and $F_{\alpha 0} \in \mathcal{S}_{\alpha}$ be the initial data. We denote by $\left(l_{\alpha}, u_{\alpha}\right)$ the limit of sequences $\left(l_{\alpha}^{0}, u_{\alpha}^{0}\right) \in \mathcal{S}_{\alpha, T}$ satisfying the beginning condition. Then we have

$$
l_{A}=u_{A}, \quad l_{B}=u_{B} .
$$

Moreover, the common limit is a mild solution to (1.1).

Proof. Consider the equation for $u_{\alpha}-l_{\alpha}, \alpha \in\{A, B\}, \quad \alpha \neq \beta$ :

$$
\begin{aligned}
u_{\alpha}- & l_{\alpha} \\
= & \int_{0}^{t}\left[Q_{+}^{\alpha{ }^{\alpha \sharp}}\left(u_{\alpha}-l_{\alpha}, l_{\alpha}\right)+Q_{+}^{\alpha \alpha \sharp}\left(l_{\alpha}, u_{\alpha}-l_{\alpha}\right)-\left(u_{\alpha}-l_{\alpha}\right) R^{\alpha \alpha \sharp}\left(l_{\alpha}\right)+l_{\alpha} R^{\alpha{ }^{\prime}}\left(u_{\alpha}-l_{\alpha}\right)\right. \\
& \left.+Q_{+}^{\alpha \beta \sharp}\left(u_{\alpha}-l_{\alpha}, l_{\beta}\right)+Q_{+}^{\alpha \beta \sharp}\left(l_{\alpha}, u_{\beta}-l_{\beta}\right)-\left(u_{\alpha}-l_{\alpha}\right) R^{\alpha \beta \sharp}\left(l_{\beta}\right)+l_{\alpha} R^{\alpha \beta \sharp}\left(u_{\beta}-l_{\beta}\right)\right] d s .
\end{aligned}
$$

It follows from Lemma 3.3 that

$$
\begin{array}{rl}
\left|u_{\alpha}-l_{\alpha}\right| \leq 4\left(C_{\alpha \alpha}+C_{\alpha \beta}\right)[ & \left\|u_{\alpha}-l_{\alpha}\right\|_{\alpha}\left\|l_{\alpha}\right\|_{\alpha}+\left\|l_{\alpha}\right\|_{\alpha}\left\|u_{\alpha}-l_{\alpha}\right\|_{\alpha} \\
& \left.+\left\|u_{\alpha}-l_{\alpha}\right\|_{\alpha}\left\|l_{\beta}\right\|_{\beta}+\left\|l_{\alpha}\right\|_{\alpha}\left\|u_{\beta}-l_{\beta}\right\|_{\beta}\right] M_{\alpha} \\
\leq 4 & 4\left(C_{\alpha \alpha}+C_{\alpha \beta}\right)\left(\delta_{\alpha}+\delta_{\beta}\right)\left[\left\|u_{\alpha}-l_{\alpha}\right\|_{\alpha}+\left\|u_{\beta}-l_{\beta}\right\|_{\beta}\right] M_{\alpha} .
\end{array}
$$

Hence we have

$$
\left\|u_{\alpha}-l_{\alpha}\right\| \leq 4\left(C_{\alpha \alpha}+C_{\alpha \beta}\right)\left(\delta_{\alpha}+\delta_{\beta}\right)\left[\left\|u_{\alpha}-l_{\alpha}\right\|_{\alpha}+\left\|u_{\beta}-l_{\beta}\right\|_{\beta}\right] .
$$


This yields

$$
\left\|u_{A}-l_{A}\right\|+\left\|u_{B}-l_{B}\right\| \leq 4 \sum_{\alpha, \beta \in\{A, B\}}\left(C_{\alpha \alpha}+C_{\alpha \beta}\right)\left(\delta_{A}+\delta_{B}\right)\left[\left\|u_{A}-l_{A}\right\|_{A}+\left\|u_{B}-l_{B}\right\|_{B}\right] .
$$

Hence for sufficiently small $\delta_{A}, \delta_{B}$, we have $u_{A}=l_{A}, u_{B}=l_{B}$. It suffices to show that these limits are mild solutions. We integrate (3.5) to get

$$
\begin{aligned}
l_{A}^{(k+1) \sharp}(x, \xi, t)=F_{A 0}(x, \xi)+\int_{0}^{t} & {\left[Q_{+}^{A A \sharp}\left(l_{A}^{k}, l_{A}^{k}\right)+Q_{+}^{A B \sharp}\left(l_{A}^{k}, l_{B}^{k}\right)\right](x, \xi, \tau) } \\
& -l_{A}^{(k+1) \sharp}(x, \xi, \tau)\left[R^{A A \sharp}\left(u_{A}^{k}\right)+R^{A B \sharp}\left(u_{B}^{k}\right)\right](x, \xi, \tau) d \tau, \\
l_{B}^{(k+1) \sharp}(x, \xi, t)=F_{B 0}(x, \xi)+\int_{0}^{t} & {\left[Q_{+}^{B B \sharp}\left(l_{B}^{k}, l_{B}^{k}\right)+Q_{+}^{B A \sharp}\left(l_{B}^{k}, l_{A}^{k}\right)\right](x, \xi, \tau) } \\
& -l_{B}^{(k+1) \sharp}(x, \xi, \tau)\left[R^{B B \sharp}\left(u_{B}^{k}\right)+R^{B A \sharp}\left(u_{A}^{k}\right)\right](x, \xi, \tau) d \tau .
\end{aligned}
$$

Because we know $\left\{l_{\alpha}^{k}\right\}$ is a bounded increasing sequence, we can pass to the limit in the integrals and $l_{\alpha}=u_{\alpha}=F_{\alpha}$ become mild solutions to the system (1.1).

Hence it suffices to find suitable functions $u_{A}^{0}(t), u_{B}^{0}(t), l_{A}^{0}(t), l_{B}^{0}(t)$ satisfying the beginning condition globally to close the Kaniel-Shinbrot argument.

The proof of Theorem 2.1 (existence part). We set

$$
l_{A}^{0}=0, \quad l_{B}^{0}=0, \quad u_{A}^{0}=2 \delta_{A} e^{-m_{A}\left(p|x|^{2}+q|\xi|^{2}\right)} \quad \text { and } \quad u_{B}^{0}=2 \delta_{B} e^{-m_{B}\left(p|x|^{2}+q|\xi|^{2}\right)} .
$$

Then it follows from (3.8) that

$$
l_{\alpha}^{1}=F_{\alpha 0} e^{-\int_{0}^{t}\left[R^{\alpha \alpha \sharp}\left(u_{\alpha}^{0}\right)+R^{\alpha \beta \sharp}\left(u_{\beta}^{0}\right)\right] d s},
$$

and it is obvious to see that

$$
l_{\alpha}^{1} \geq l_{\alpha}^{0}=0 .
$$

Similarly, $u_{\alpha}^{1}$ can be constructed and

$$
u_{\alpha}^{1}=F_{\alpha 0}+\int_{0}^{t}\left[Q_{+}^{\alpha \alpha \sharp}\left(u_{\alpha}^{0}, u_{\alpha}^{0}\right)+Q_{+}^{\alpha \beta \sharp}\left(u_{\alpha}^{0}, u_{\beta}^{0}\right)\right] d s,
$$

which shows that $u_{\alpha}^{1} \geq l_{\alpha}^{1}$ and, from Lemma 3.2,

$$
\begin{aligned}
u_{\alpha}^{1} & =F_{\alpha 0}+\int_{0}^{t}\left[Q_{+}^{\alpha \alpha \sharp}\left(u_{\alpha}^{0}, u_{\alpha}^{0}\right)+Q_{+}^{\alpha \beta \sharp}\left(u_{\alpha}^{0}, u_{\beta}^{0}\right)\right] d s \\
& \leq \delta_{\alpha}\left(1+4 C_{\alpha \alpha} \delta_{\alpha}+4 C_{\alpha \beta} \delta_{\beta}\right) e^{-m_{\alpha}\left(p|x|^{2}+q|\xi|^{2}\right)} .
\end{aligned}
$$

Since

$$
\delta_{\alpha}, \delta_{\beta} \leq\left(4 \sum_{\alpha, \beta \in\{A, B\}}\left(C_{\alpha \alpha}+C_{\alpha \beta}\right)\right)^{-1}
$$

we have

$$
u_{\alpha}^{1} \leq 2 \delta_{\alpha} e^{-m_{\alpha}\left(p|x|^{2}+q|\xi|^{2}\right)}=u_{\alpha}^{0} .
$$

Hence we have shown that the beginning condition holds.

REMARK 3.1. The uniqueness of a mild solution can be shown by the uniform $L^{1}$ stability estimate in Theorem 1.3. 
4. Approximate Boltzmann system. In this section, we review the mollification procedure in [13, 19] to get smooth approximate solutions to the Boltzmann system (1.1). Since the time-evolution estimates for the nonlinear functionals along the solutions in the following sections rely on the differentiability of the solution, we cannot apply the timedecay analysis to the continuous mild solutions directly. Hence, we need to mollify our mild solutions constructed in the previous section and obtain the time-evolution estimates for the nonlinear functionals along the smooth approximate solutions, and then we pass to the limit to get the desired result. The detailed explanation can be found in the aforementioned two references [13, 19. For simplicity of presentation, we suppress $t$ dependence in $F$ and $Q(F, F)$ :

$$
F(x, \xi):=F(x, \xi, t) \quad \text { and } \quad Q(F, F)(x, \xi):=Q(F, F)(x, \xi, t) .
$$

Let $\phi \in C_{c}^{\infty}\left(\mathbb{R}^{3}\right)$ and $\phi_{\varepsilon}$ be the standard mollifier and a corresponding rescaled mollifier respectively, i.e.,

$$
\begin{aligned}
& 0 \leq \phi \leq 1, \quad \operatorname{supp}(\phi) \subset B_{1}(0), \quad \int_{\mathbb{R}^{3}} \phi(x) d x=1, \\
& \phi_{\varepsilon}(x):=\frac{1}{\varepsilon^{3}} \phi\left(\frac{x}{\varepsilon}\right), \quad \operatorname{supp}\left(\phi_{\varepsilon}\right) \subset B_{\varepsilon}(0), \quad x \in \mathbb{R}^{3}, \varepsilon>0,
\end{aligned}
$$

where $B_{\varepsilon}(0)$ is the ball with a radius $\varepsilon$ centered at the origin. We denote the mollifications of $F_{\alpha}$ and $Q^{\alpha \beta}\left(F_{\alpha}, F_{\beta}\right)$ as

$$
F_{\alpha \varepsilon}:=F_{\alpha} *_{x} \phi_{\varepsilon} \quad \text { and } \quad Q_{\varepsilon}^{\alpha \beta}\left(F_{\alpha}, F_{\beta}\right):=Q^{\alpha \beta}\left(F_{\alpha}, F_{\beta}\right) *_{x} \phi_{\varepsilon},
$$

where $*_{x}$ denotes a convolution with respect to the $x$-variable.

Consider the mollified Boltzmann system:

$$
\begin{aligned}
\partial_{t} F_{A \varepsilon}+\xi \cdot \nabla_{x} F_{A \varepsilon} & =Q^{A A}\left(F_{A \varepsilon}, F_{A \varepsilon}\right)+Q^{A B}\left(F_{A \varepsilon}, F_{B \varepsilon}\right)+P_{\varepsilon}^{A A}+P_{\varepsilon}^{A B}, \\
\partial_{t} F_{B \varepsilon}+\xi \cdot \nabla_{x} F_{B \varepsilon} & =Q^{B B}\left(F_{B \varepsilon}, F_{B \varepsilon}\right)+Q^{B A}\left(F_{B \varepsilon}, F_{A \varepsilon}\right)+P_{\varepsilon}^{B B}+P_{\varepsilon}^{B A}, \\
F_{A \varepsilon}(x, \xi, 0) & =F_{A 0} *_{x} \phi_{\varepsilon}, \quad F_{B \varepsilon}(x, \xi, 0)=F_{B 0} *_{x} \phi_{\varepsilon} .
\end{aligned}
$$

Here $P_{\varepsilon}^{\alpha \beta}$ represents the perturbation given by

$$
\begin{aligned}
& P_{+\varepsilon}^{\alpha \beta}:=Q_{+\varepsilon}^{\alpha \beta}\left(F_{\alpha}, F_{\beta}\right)-Q_{+}^{\alpha \beta}\left(F_{\alpha \varepsilon}, F_{\beta \varepsilon}\right), \\
& P_{-\varepsilon}^{\alpha \beta}:=Q_{-\varepsilon}^{\alpha \beta}\left(F_{\alpha}, F_{\beta}\right)-Q_{-}^{\alpha \beta}\left(F_{\alpha \varepsilon}, F_{\beta \varepsilon}\right), \\
& P_{\varepsilon}^{\alpha \beta}:=P_{+\varepsilon}^{\alpha \beta}-P_{-\varepsilon}^{\alpha \beta}, \quad \alpha, \beta \in\{A, B\} .
\end{aligned}
$$

Moreover, we also define a notation:

$$
P(x, \xi, \varepsilon):=\sum_{\alpha, \beta \in\{A, B\}}\left|P_{\varepsilon}^{\alpha \beta}\right| .
$$

Below we list several lemmas which can be obtained from the similar arguments used in [19]. From now on, $C$ represents a generic constant independent of the mollification parameter $\varepsilon$ and time $t$.

Lemma 4.1 (19]). Let $\left(F_{A}, F_{B}\right)$ be mild solutions in Theorem 2.1. Then we have

$$
F_{\alpha \varepsilon}^{\sharp}(x, \xi) \leq \delta_{\alpha} M_{\alpha \varepsilon}(x, \xi) \quad \text { and } \quad M_{\alpha \varepsilon}(x, \xi) \leq e^{m_{\alpha} p} e^{-m_{\alpha}\left(\frac{p}{2}|x|^{2}+q|\xi|^{2}\right)}, \quad \alpha \in\{A, B\} .
$$


Lemma $4.2([19])$. Let $M_{\alpha}$ and $M_{\alpha \varepsilon}$ be a local maxwellian and its mollification. Then we have

$$
\begin{gathered}
\int_{\mathbb{R}^{3} \times \mathbb{R}^{+}}\left|\xi-\xi_{*}\right| M_{\alpha \varepsilon}\left(x+t\left(\xi-\xi_{*}\right)+\tau\left(\xi-\xi_{*}\right), \xi_{*}\right) d \tau d \xi_{*} \leq C_{1}^{\alpha} \delta_{\alpha}, \\
\int_{\mathbb{R}^{3} \times \mathbf{S}^{2}}\left|\xi-\xi_{*}\right| M_{\alpha \varepsilon}\left(x+t\left(\xi-\xi_{*}\right), \xi_{*}\right) d \omega d \xi_{*} \leq C_{2}^{\alpha} \delta_{\alpha} \frac{(1+|x|+|\xi|)}{(t+1)^{4}}
\end{gathered}
$$

where we set

$$
\begin{aligned}
& C_{1}^{\alpha}:=2 \pi e^{m_{\alpha} p}\left(\frac{2 \pi}{p m_{\alpha}}\right)^{\frac{1}{2}}\left(\frac{\pi}{q m_{\alpha}}\right)^{\frac{3}{2}}, \\
& C_{2}^{\alpha}:=16 \pi e^{m_{\alpha} p}\left[\left(\frac{\pi}{m_{\alpha} p}\right)^{\frac{3}{2}}+\frac{2 \pi}{\left(m_{\alpha} p\right)^{2}}+\left(\frac{\pi}{m_{\alpha} q}\right)^{\frac{3}{2}}+\frac{2 \pi}{\left(m_{\alpha} q\right)^{2}}\right] .
\end{aligned}
$$

Lemma 4.3. Let $F_{\alpha}$ be a mild solution to (1.1) with initial datum $F_{\alpha 0}$ in Theorem 2.1. Then we have

$$
\left(Q_{+}^{\alpha \beta \sharp}\left(F_{\alpha \varepsilon}, F_{\beta \varepsilon}\right)+Q_{-}^{\alpha \beta \sharp}\left(F_{\alpha \varepsilon}, F_{\beta \varepsilon}\right)\right)(x, \xi) \leq C_{1}^{\alpha \beta} \delta_{\alpha} \delta_{\beta} \frac{e^{-m_{\alpha}\left(\frac{p}{3}|x|^{2}+\frac{q}{2}|\xi|^{2}\right)}}{(t+1)^{4}} .
$$

Here the positive constant $C_{1}^{\alpha \beta}$ is defined by

$$
C_{1}^{\alpha \beta}:=e^{m_{\alpha} p}\left\|A_{\alpha \beta}\right\|_{\infty} C_{2}^{\beta} \sup _{x, \xi}\left\{(1+|x|+|\xi|) e^{-m_{\beta} \frac{p}{6}|x|^{2}-m_{\beta} \frac{q}{6}|\xi|^{2}}\right\} .
$$

Proof. We only consider the pointwise estimate for the gain operator. The estimate for the loss operator can be treated similarly. Recall the pointwise estimate for $F$ :

$$
F_{\alpha \varepsilon}^{\sharp}(x, \xi) \leq \delta_{\alpha} M_{\alpha \varepsilon}(x, \xi) .
$$

Then we use the above pointwise estimate together with Lemma 3.2 and 4.2 to see that

$$
\begin{aligned}
& Q_{+}^{\alpha \beta \sharp}\left(F_{\alpha \varepsilon}, F_{\beta \varepsilon}\right)(x, \xi) \\
& \quad=\int_{\mathbb{R}^{3} \times \mathbf{S}^{2}} \frac{\left|\left(\xi-\xi_{*}\right) \cdot \omega\right|}{e^{\prime} J^{\prime}} F_{\alpha \varepsilon}^{\sharp}\left(x+t\left(\xi-\xi^{\prime}\right), \xi^{\prime}\right) F_{\beta \varepsilon}^{\sharp}\left(x+t\left(\xi-\xi_{*}^{\prime}\right), \xi_{*}^{\prime}\right) d \omega d \xi_{*} \\
& \quad \leq \delta_{\alpha} \delta_{\beta} \int_{\mathbb{R}^{3} \times \mathbf{S}^{2}} \frac{\left|\left(\xi-\xi_{*}\right) \cdot \omega\right|}{e^{\prime} J^{\prime}} M_{\alpha \varepsilon}\left(x+t\left(\xi-\xi^{\prime}\right), \xi^{\prime}\right) M_{\beta \varepsilon}\left(x+t\left(\xi-\xi_{*}^{\prime}\right), \xi_{*}^{\prime}\right) d \omega d \xi_{*} \\
& \quad \leq e^{p\left(m_{\alpha}+m_{\beta}\right)} \delta_{\alpha} \delta_{\beta} e^{-m_{\alpha}\left(\frac{p}{2}|x|^{2}+q|\xi|^{2}\right)} \\
& \quad \times \int_{\mathbb{R}^{3} \times \mathbf{S}^{2}} \frac{\left|\left(\xi-\xi_{*}\right) \cdot \omega\right|}{e^{\prime} J^{\prime}} e^{-m_{\beta}\left(\frac{p}{2}\left|x+t\left(\xi-\xi_{*}\right)\right|^{2}+q\left|\xi_{*}\right|^{2}\right)} e^{-\left(\frac{p}{2} t^{2}+q\right) \frac{m_{\alpha} m_{\beta}}{m_{\alpha}+m_{\beta}}\left(1-e^{\prime 2}\right)\left(\left(\xi^{\prime}-\xi_{*}^{\prime}\right) \cdot \omega\right)^{2}} d \omega d \xi_{*} \\
& \leq 2 \pi e^{p\left(m_{\alpha}+m_{\beta}\right)}\left\|A_{\alpha \beta}\right\|_{\infty} \delta_{\alpha} \delta_{\beta} e^{-m_{\alpha}\left(\frac{p}{2}|x|^{2}+q|\xi|^{2}\right)} \int_{\mathbb{R}^{3}}\left|\xi-\xi_{*}\right| e^{-m_{\beta}\left(\frac{p}{2}\left|x+t\left(\xi-\xi_{*}\right)\right|^{2}+q\left|\xi_{*}\right|^{2}\right)} d \xi_{*} \\
& \leq C_{1}^{\alpha \beta} \delta_{\alpha} \delta_{\beta} \frac{e^{-m_{\alpha}\left(\frac{p}{3}|x|^{2}+\frac{q}{2}|\xi|^{2}\right)}}{(t+1)^{4}} .
\end{aligned}
$$

Lemma 4.4. The time-phase space integral of the perturbation term (4.3) vanishes as $\varepsilon \rightarrow 0$, i.e.,

$$
\lim _{\varepsilon \rightarrow 0} \int_{0}^{t} \int_{\mathbb{R}^{6}}(1+|\xi|) P^{\sharp}(x, \xi, \varepsilon) d \xi d x d s=0 .
$$


Proof. It suffices to show that

$$
\lim _{\varepsilon \rightarrow 0} \int_{0}^{t} \int_{\mathbb{R}^{6}}\left((1+|\xi|)\left|P_{+}^{\alpha \beta \sharp}\right|+(1+|\xi|)\left|P_{-}^{\alpha \beta \sharp}\right|\right) d \xi d x d s=0 .
$$

We first note the elementary property of mollification:

$$
\begin{aligned}
& Q_{-\varepsilon}^{\alpha \beta}\left(F_{\alpha}, F_{\beta}\right) \rightarrow Q_{-}^{\alpha \beta}\left(F_{\alpha}, F_{\beta}\right) \quad \text { a.e }(x, \xi) \quad \text { as } \varepsilon \rightarrow 0, \\
& \frac{\left|\left(\xi-\xi_{*}\right) \cdot \omega\right|}{e^{\prime} J^{\prime}} F_{\alpha \varepsilon}^{\prime \sharp} F_{\beta * \varepsilon}^{\prime \sharp} \rightarrow \frac{\left|\left(\xi-\xi_{*}\right) \cdot \omega\right|}{e^{\prime} J^{\prime}} F_{\alpha}^{\prime \sharp} F_{\beta *}^{\prime \sharp}, \quad \text { a.e }(x, \xi) \quad \text { as } \varepsilon \rightarrow 0 .
\end{aligned}
$$

Then the above relation, Lemma 4.1, and (C) imply

$$
\begin{aligned}
& \frac{\left|\left(\xi-\xi_{*}\right) \cdot \omega\right|}{e^{\prime} J^{\prime}} F_{\alpha \varepsilon}^{\sharp} F_{\beta * \varepsilon}^{\sharp} \\
& \leq e^{\left(m_{\alpha}+m_{\beta}\right) p} e^{-m_{\alpha} \frac{p}{2}|x|^{2}} e^{-m_{\beta} \frac{p}{2}\left|x+t\left(\xi-\xi_{*}\right)\right|^{2}} e^{-m_{\alpha} q|\xi|^{2}} e^{-m_{\beta} q\left|\xi_{*}\right|^{2}} \\
& \quad \times \frac{\left|\left(\xi-\xi_{*}\right) \cdot \omega\right|}{e^{\prime} J^{\prime}} e^{-q \frac{m_{\alpha}+m_{\beta}}{m_{\alpha} m_{\beta}}(1+e)(1-e)\left(\left(\xi-\xi_{*}\right) \cdot \omega\right)} \in L^{1}\left(\mathbb{R}_{\xi_{*}}^{3} \times \mathbf{S}^{2}\right) .
\end{aligned}
$$

We now apply the Lebesgue dominated convergence theorem (LDCT in short) to find

$$
Q_{+}^{\alpha \beta \sharp}\left(F_{\alpha \varepsilon}, F_{\beta \varepsilon}\right) \rightarrow Q_{+}^{\alpha \beta \sharp}\left(F_{\alpha}, F_{\beta}\right), \quad \text { a.e }(x, \xi) \quad \text { as } \quad \varepsilon \rightarrow 0 .
$$

We combine the above estimate with (4.5) to get

$$
P_{+\varepsilon}^{\alpha \beta}=Q_{+\varepsilon}^{\alpha \beta}\left(F_{\alpha}, F_{\beta}\right)-Q_{+}^{\alpha \beta}\left(F_{\alpha \varepsilon}, F_{\beta \varepsilon}\right) \rightarrow 0, \quad \text { a.e }(x, \xi) \quad \text { as } \quad \varepsilon \rightarrow 0
$$

or equivalently,

$$
(1+|\xi|) P_{+\varepsilon}^{\alpha \beta} \rightarrow 0, \quad \text { a.e }(x, \xi) \quad \text { as } \quad \varepsilon \rightarrow 0 .
$$

On the other hand, it follows from Lemma 4.2 that

$$
\begin{aligned}
(1+|\xi|)\left|P_{+\varepsilon}^{\alpha \beta}\right| \leq & (1+|\xi|)\left|Q_{+\varepsilon}^{\alpha \beta \sharp}\left(F_{\alpha}, F_{\alpha}\right)\right|+(1+|\xi|)\left|Q_{+}^{\alpha \beta \sharp}\left(F_{\alpha \varepsilon}, F_{\alpha \varepsilon}\right)\right| \\
& \leq C(1+|\xi|)\left(\frac{e^{-m_{\alpha}\left(\frac{p}{2}|x|^{2}+\frac{q}{2}|\xi|^{2}\right)}}{(t+1)^{4}}\right)_{\varepsilon}+C(1+|\xi|) \frac{\left.e^{-m_{\alpha}\left(\frac{p}{3}|x|^{2}+\frac{q}{2}|\xi|^{2}\right.}\right)}{(t+1)^{4}} \\
& \leq C \frac{e^{-m_{\alpha}\left(\frac{p}{3}|x|^{2}+\frac{q}{3}|\xi|^{2}\right)}}{(t+1)^{4}} \in L^{1}\left(\mathbb{R}_{x}^{3} \times \mathbb{R}_{\xi}^{3} \times \mathbb{R}_{+}\right)
\end{aligned}
$$

and we apply the LDCT again to see

$$
\lim _{\varepsilon \rightarrow 0} \int_{0}^{t} \int_{\mathbb{R}^{6}}(1+|\xi|)\left|P_{+\varepsilon}^{\alpha \beta \sharp}\right| d \xi d x d s=0 .
$$

Similarly, we have

$$
\lim _{\varepsilon \rightarrow 0} \int_{0}^{t} \int_{\mathbb{R}^{6}}(1+|\xi|)\left|P_{-\varepsilon}^{\alpha \beta \sharp}\right| d \xi d x d s=0 .
$$

In the following three sections, we introduce several nonlinear functionals along the approximate solutions, and via the time-evolution estimates of functionals we prove that the mild solutions constructed in Section 3 satisfy scattering and uniform $L^{1}$-type stability estimates. Moreover, we also provide new a priori estimates without any smallness assumptions. 
5. Generalized collision potential. In this section, we introduce a generalized collision potential introduced in [17, 19] and as a direct application, we see the large time behavior of mild solutions constructed in Section 3.

To incorporate the weight $\varphi_{\alpha, r}=\left(1+m_{\alpha}|\xi|^{2}\right)^{\frac{r}{2}}$ in the velocity space, we set

$$
f_{A}:=\varphi_{\alpha, r} F_{A}, \quad f_{B}:=\varphi_{\alpha, r} F_{B} .
$$

Then the $L_{r}^{1}$-distance of $F_{A}$ and $F_{B}$ can be viewed as the $L^{1}$-distance of $f_{A}$ and $f_{B}$. Recall that $f_{A}$ and $f_{B}$ satisfy

$$
\begin{aligned}
& \partial_{t} f_{A}+\xi \cdot \nabla_{x} f_{A}=\mathcal{Q}^{A A}\left(f_{A}, f_{A}\right)+\mathcal{Q}^{A B}\left(f_{A}, f_{B}\right), \quad x, \xi \in \mathbb{R}^{3}, t>0, \\
& \partial_{t} f_{B}+\xi \cdot \nabla_{x} f_{B}=\mathcal{Q}^{B B}\left(f_{B}, f_{B}\right)+\mathcal{Q}^{B A}\left(f_{B}, f_{A}\right), \\
& \left(f_{A}, f_{B}\right)(x, \xi, 0)=\left(f_{A 0}, f_{B 0}\right)(x, \xi),
\end{aligned}
$$

where the collision operator for $f_{A}$ and $f_{B}$ can be rewritten as:

$$
\begin{aligned}
& \mathcal{Q}^{\alpha \beta}\left(f_{\alpha}, f_{\beta}\right)(\xi) \\
& \quad:=\int_{\mathbb{R}^{3} \times \mathbf{S}^{2}}\left|\left(\xi-\xi_{*}\right) \cdot \omega\right|\left(\frac{\varphi_{\alpha, r}(\xi)}{\varphi_{\alpha, r}\left(\xi^{\prime}\right) \varphi_{\beta, r}\left(\xi_{*}^{\prime}\right)} \frac{f_{\alpha}\left(\xi^{\prime}\right) f_{\beta}\left(\xi_{*}^{\prime}\right)}{e^{\prime} J^{\prime}}-\frac{1}{\varphi_{\beta, r}\left(\xi_{*}\right)} f_{\alpha}(\xi) f_{\beta}\left(\xi_{*}\right)\right) d \omega d \xi_{*} .
\end{aligned}
$$

Following the mollification procedure introduced in the previous section, we mollify $f_{A}$ and $f_{B}$, and then their mollifiers $f_{A \varepsilon}$ and $f_{B \varepsilon}$ satisfy

$$
\begin{aligned}
& \partial_{t} f_{A \varepsilon}+\xi \cdot \nabla_{x} f_{A \varepsilon}=\mathcal{Q}^{A A}\left(f_{A \varepsilon}, f_{A \varepsilon}\right)+\mathcal{Q}^{A B}\left(f_{A \varepsilon}, f_{B \varepsilon}\right)+\mathcal{P}_{\varepsilon}^{A A}+\mathcal{P}_{\varepsilon}^{A B}, \\
& \partial_{t} f_{B \varepsilon}+\xi \cdot \nabla_{x} f_{B \varepsilon}=\mathcal{Q}^{B B}\left(f_{B \varepsilon}, f_{B \varepsilon}\right)+\mathcal{Q}^{B A}\left(f_{B \varepsilon}, f_{A \varepsilon}\right)+\mathcal{P}_{\varepsilon}^{B B}+\mathcal{P}_{\varepsilon}^{B A}, \\
& f_{A \varepsilon}(x, \xi, 0)=f_{A 0} *_{x} \phi_{\varepsilon}, \quad f_{B \varepsilon}(x, \xi, 0)=f_{B 0} *_{x} \phi_{\varepsilon},
\end{aligned}
$$

where $\mathcal{P}_{\varepsilon}^{\alpha \beta}$ is given by

$$
\begin{aligned}
\mathcal{P}_{ \pm \varepsilon}^{\alpha \beta} & :=\mathcal{Q}_{ \pm \varepsilon}^{\alpha \beta}\left(f_{\alpha}, f_{\beta}\right)-\mathcal{Q}_{ \pm}^{\alpha \beta}\left(f_{\alpha \varepsilon}, f_{\beta \varepsilon}\right), \\
\mathcal{P}_{\varepsilon}^{\alpha \beta} & :=\mathcal{P}_{+\varepsilon}^{\alpha \beta}-\mathcal{P}_{-\varepsilon}^{\alpha \beta}, \quad \alpha, \beta \in\{A, B\}, \\
\mathcal{P}(x, \xi, \varepsilon) & :=\sum_{\alpha, \beta \in\{A, B\}}\left|\mathcal{P}_{\varepsilon}^{\alpha \beta}\right| .
\end{aligned}
$$

Next lemma shows that these mollified collision operators for $\left(f_{A}, f_{B}\right)$ are dominated by the original one and the time-phase space integral of perturbation in (5.4) vanishes as $\varepsilon \rightarrow 0$.

Lemma 5.1. $Q^{\alpha \beta}\left(f_{\alpha}, f_{\beta}\right)$ and $\mathcal{P}_{ \pm \varepsilon}^{\alpha \beta}$ satisfy

$$
\begin{aligned}
& \text { (i) }\left|\mathcal{Q}_{ \pm}^{\alpha \beta}\left(f_{\alpha}, f_{\beta}\right)(x, \xi)\right| \leq\left|Q_{ \pm}^{\alpha \beta}\left(f_{\alpha}, f_{\beta}\right)(\xi)\right| . \\
& \text { (ii) }\left|\mathcal{P}_{ \pm \varepsilon}^{\alpha \beta}(x, \xi)\right| \leq\left|Q_{ \pm \varepsilon}^{\alpha \beta}\left(f_{\alpha}, f_{\beta}\right)(x, \xi)\right|+\left|Q_{ \pm}^{\alpha \beta}\left(f_{\alpha \varepsilon}, f_{\beta \varepsilon}\right)(x, \xi)\right| . \\
& \text { (iii) } \lim _{\varepsilon \rightarrow 0} \int_{0}^{t} \int_{\mathbb{R}^{6}} \mathcal{P}(x, \xi, \varepsilon) d x d \xi d s=0 .
\end{aligned}
$$

Proof. (1) We use $\frac{1}{\varphi_{\beta, r}\left(\xi_{*}\right)} \leq 1$ and energy dissipation to show that

$$
\frac{\varphi_{\alpha, r}(\xi)}{\varphi_{\alpha, r}\left(\xi^{\prime}\right) \varphi_{\beta, r}\left(\xi_{*}^{\prime}\right)} \leq\left(\frac{1+m_{\alpha}|\xi|^{2}}{\left(1+m_{\alpha}\left|\xi^{\prime}\right|^{2}\right)\left(1+m_{\beta}\left|\xi_{*}^{\prime}\right|^{2}\right)}\right)^{\frac{r}{2}}
$$




$$
\begin{aligned}
& \leq\left(\frac{1+m_{\alpha}|\xi|^{2}}{1+m_{\alpha}\left|\xi^{\prime}\right|^{2}+m_{\beta}\left|\xi_{*}^{\prime}\right|+m_{\alpha} m_{\beta}\left|\xi^{\prime}\right|^{2}\left|\xi_{*}^{\prime}\right|^{2}}\right)^{\frac{r}{2}} \\
& \leq\left(\frac{1+m_{\alpha}|\xi|^{2}}{1+m_{\alpha}|\xi|^{2}+m_{\beta}\left|\xi_{*}\right|+m_{\alpha} m_{\beta}\left|\xi^{\prime}\right|^{2}\left|\xi_{*}^{\prime}\right|^{2}}\right)^{\frac{r}{2}} \leq 1 .
\end{aligned}
$$

It is easy to see that (2) directly follows from (1) and with (1) and (2), the proof of (3) can be treated the same as the proof of Lemma 4.4.

5.1. Collision potential. We now define the collision potentials for $f_{\varepsilon}=\left(f_{A \varepsilon}, f_{B \varepsilon}\right)$ corresponding to the mollified mild solutions $F_{A \varepsilon}, F_{B \varepsilon}$ as follows:

$$
\begin{aligned}
& \mathcal{D}^{\alpha \beta}\left(f_{\varepsilon}(t)\right):=\int_{\mathbb{R}^{6}} f_{\alpha \varepsilon}^{\sharp}(x, \xi)\left[\int_{\mathbb{R}^{3}} \int_{0}^{\infty} \mid \xi\right.-\xi_{*} \mid \\
&\left.\times f_{\beta \varepsilon}^{\sharp}\left(x+(t+\tau)\left(\xi-\xi_{*}\right), \xi_{*}\right) d \tau d \xi_{*}\right] d \xi d x, \\
& \Lambda^{\alpha \beta}\left(f_{\varepsilon}(t)\right):=\int_{\mathbb{R}^{9}}\left|\xi-\xi_{*}\right| f_{\alpha \varepsilon}^{\sharp}(x, \xi) f_{\beta \varepsilon}^{\sharp}\left(x+t\left(\xi-\xi_{*}\right), \xi_{*}\right) d \xi_{*} d \xi d x, \\
& \mathcal{D}\left(f_{\varepsilon}(t)\right):=\sum_{\alpha, \beta \in\{A, B\}} \mathcal{D}^{\alpha \beta}\left(f_{\varepsilon}(t)\right), \quad \Lambda\left(f_{\varepsilon}(t)\right):=\sum_{\alpha, \beta \in\{A, B\}} \Lambda^{\alpha \beta}\left(f_{\varepsilon}(t)\right) .
\end{aligned}
$$

We now set

$$
\mathcal{D}(f(t)):=\lim _{\varepsilon \rightarrow 0} \mathcal{D}\left(f_{\varepsilon}(t)\right), \quad \Lambda(f(t)):=\lim _{\varepsilon \rightarrow 0} \Lambda\left(f_{\varepsilon}(t)\right) .
$$

Lemma 5.2. The phase-space integral of collision operators is bounded by the collision production rate $\Lambda^{\alpha \beta}\left(f_{\varepsilon}(t)\right)$ :

$$
\int_{\mathbb{R}^{6}}\left(\mathcal{Q}_{+\varepsilon}^{\alpha \beta \sharp}\left(f_{\alpha}, f_{\beta}\right)+\mathcal{Q}_{-\varepsilon}^{\alpha \beta \sharp}\right)\left(f_{\alpha}, f_{\beta}\right) d \xi d x \leq C \Lambda^{\alpha \beta}\left(f_{\varepsilon}(t)\right) .
$$

Proof. We use the change of variables $\left(\xi^{\prime}, \xi_{*}^{\prime}\right) \leftrightarrow\left(\xi, \xi_{*}\right)$ and the Fubini theorem to see

$$
\begin{aligned}
\int_{\mathbb{R}^{6}}\left(\mathcal { Q } _ { + \varepsilon } ^ { \alpha \beta \sharp } \left(f_{\alpha},\right.\right. & \left.\left.f_{\beta}\right)+\mathcal{Q}_{-\varepsilon}^{\alpha \beta \sharp}\left(f_{\alpha}, f_{\beta}\right)\right) d \xi d x \\
& \leq \int_{\mathbb{R}^{9} \times \mathbf{S}^{2}}\left|\xi-\xi_{*}\right|\left(\frac{f_{\alpha \varepsilon}^{\prime} f_{\beta * \varepsilon}^{\prime}}{e^{\prime} J^{\prime}}+f_{\alpha \varepsilon} f_{\beta * \varepsilon}\right) d \omega d \xi_{*} d \xi d x \\
& \leq 8 \pi \int_{\mathbb{R}^{9}}\left|\xi-\xi_{*}\right| f_{\alpha \varepsilon} f_{\beta * \varepsilon} d \xi_{*} d \xi d x \\
& \leq 8 \pi \Lambda^{\alpha \beta}\left(f_{\varepsilon}(t)\right) .
\end{aligned}
$$

5.2. Time-decay estimate. In this part, we study the time-decay estimate of the nonlinear functional introduced in the previous subsection. The functional does not have pairs of particles that have just finished collisions due to its construction. Moreover, in the construction, we used the future density of the particles along the forward particle trajectories by the present density for a given time; hence, this will generate errors involving the time-integral $\int_{0}^{t} Q^{\sharp}(f, f) d s$. So we need to employ a kind of smallness assumption to control the cubic error terms $f \times Q(f, f)$ by the good quadratic term. The next lemma illustrates how this intuitive idea can be verified. 
Proposition 5.1. Let $f_{A}$ and $f_{B}$ be the re-scaled functions of the mild solutions $F_{A}$ and $F_{B}$ to the system (1.1) constructed in Section 3. Then $\mathcal{D}(f(t))$ satisfies

$$
\mathcal{D}(f(t))+C \int_{0}^{t} \Lambda(f(s)) d s \leq \mathcal{D}\left(f_{0}\right), \quad t \geq 0,
$$

where $C$ is a positive constant independent of time $t$.

Proof. Note that $f_{\alpha \varepsilon}^{\sharp}$ and $f_{\beta \varepsilon}^{\sharp}$ satisfy

$$
\begin{aligned}
\partial_{t} f_{\alpha \varepsilon}^{\sharp}(x, \xi)= & {\left[\mathcal{Q}^{\alpha \alpha \sharp}\left(f_{\alpha \varepsilon}, f_{\alpha \varepsilon}\right)+\mathcal{Q}^{\alpha \beta \sharp}\left(f_{\alpha \varepsilon}, f_{\beta \varepsilon}\right)\right](x, \xi) } \\
& +\left[\mathcal{P}_{\varepsilon}^{\alpha \alpha \sharp}+\mathcal{P}_{\varepsilon}^{\alpha \beta \sharp}\right](x, \xi), \\
\partial_{t}\left[f_{\beta \varepsilon}^{\sharp}(x+\right. & \left.\left.(t+\tau)\left(\xi-\xi_{*}\right), \xi_{*}\right)\right] \\
& =\partial_{\tau}\left[f_{\beta \varepsilon}^{\sharp}\left(x+(t+\tau)\left(\xi-\xi_{*}\right), \xi_{*}\right)\right] \\
& +\left[\mathcal{Q}^{\beta \beta \sharp}\left(f_{\beta \varepsilon}, f_{\beta \varepsilon}\right)+\mathcal{Q}^{\beta \alpha \sharp}\left(f_{\beta \varepsilon}, f_{\alpha \varepsilon}\right)\right]\left(x+(t+\tau)\left(\xi-\xi_{*}\right), \xi_{*}\right) \\
& +\left[\mathcal{P}_{\varepsilon}^{\beta \beta \sharp}+\mathcal{P}_{\varepsilon}^{\beta \alpha \sharp}\right]\left(x+(t+\tau)\left(\xi-\xi_{*}\right), \xi_{*}\right) .
\end{aligned}
$$

We now combine (5.5) and (5.6) to see that

$$
\begin{aligned}
\partial_{t}[\mid \xi- & \left.\xi_{*} \mid f_{\alpha \varepsilon}^{\sharp}(x, \xi) f_{\beta \varepsilon}^{\sharp}\left(x+(t+\tau)\left(\xi-\xi_{*}\right), \xi_{*}\right)\right] \\
= & \partial_{\tau}\left[\left|\xi-\xi_{*}\right| f_{\alpha \varepsilon}^{\sharp}(x, \xi) f_{\beta \varepsilon}^{\sharp}\left(x+(t+\tau)\left(\xi-\xi_{*}\right), \xi_{*}\right)\right] \\
+ & \left|\xi-\xi_{*}\right| f_{\beta \varepsilon}^{\sharp}\left(x+(t+\tau)\left(\xi-\xi_{*}\right), \xi_{*}\right) \\
& \times\left(\mathcal{Q}_{\varepsilon}^{\alpha \alpha \sharp}\left(f_{\alpha}, f_{\alpha}\right)+\mathcal{Q}_{\varepsilon}^{\alpha \beta \sharp}\left(f_{\alpha}, f_{\alpha}\right)+\mathcal{P}_{\varepsilon}^{\alpha \alpha \sharp}+\mathcal{P}_{\varepsilon}^{\alpha \beta \sharp}\right)(x, \xi) \\
+ & \left|\xi-\xi_{*}\right| f_{\alpha \varepsilon}^{\sharp}(x, \xi) \\
& \times\left(\mathcal{Q}_{\varepsilon}^{\beta \beta \sharp}\left(f_{\beta}, f_{\beta}\right)+\mathcal{Q}_{\varepsilon}^{\beta \alpha \sharp}\left(f_{\beta}, f_{\alpha}\right)+\mathcal{P}_{\varepsilon}^{\alpha \alpha \sharp}+\mathcal{P}_{\varepsilon}^{\alpha \beta \sharp}\right)\left(x+(t+\tau)\left(\xi-\xi_{*}\right), \xi_{*}\right) .
\end{aligned}
$$

We integrate (5.7) with respect to $\left(x, \xi, \xi_{*}, \tau\right) \in \mathbb{R}^{9} \times \mathbb{R}_{+}$to get

$$
\begin{aligned}
\frac{d}{d t} \mathcal{D}^{\alpha \beta}\left(f_{\varepsilon}(t)\right) & \\
= & -\int_{\mathbb{R}^{9}}\left|\xi-\xi_{*}\right| f_{\alpha \varepsilon}^{\sharp}(x, \xi) f_{\beta \varepsilon}^{\sharp}\left(x+t\left(\xi-\xi_{*}\right), \xi_{*}\right) d \xi d \xi_{*} d x \\
& +\int_{\mathbb{R}^{9} \times \mathbb{R}_{+}}\left|\xi-\xi_{*}\right| f_{\beta \varepsilon}^{\sharp}\left(x+t\left(\xi-\xi_{*}\right)+\tau\left(\xi-\xi_{*}\right), \xi_{*}\right) \\
& \quad \times\left[\mathcal{Q}_{\varepsilon}^{\alpha \alpha \sharp}\left(f_{\alpha}, f_{\alpha}\right)+\mathcal{Q}_{\varepsilon}^{\alpha \beta \sharp}\left(f_{\alpha}, f_{\alpha}\right)+\mathcal{P}_{\varepsilon}^{\alpha \alpha \sharp}+\mathcal{P}_{\varepsilon}^{\alpha \beta \sharp}\right](x, \xi) d \tau d \xi_{*} d \xi d x \\
& +\int_{\mathbb{R}^{9} \times \mathbb{R}_{+}}\left|\xi-\xi_{*}\right| f_{\alpha \varepsilon}^{\sharp}(x, \xi) \\
& \quad \times\left[\mathcal{Q}_{\varepsilon}^{\beta \beta \sharp}\left(f_{\beta}, f_{\beta}\right)+\mathcal{Q}_{\varepsilon}^{\beta \alpha \sharp}\left(f_{\beta}, f_{\alpha}\right)+\mathcal{P}_{\varepsilon}^{\beta \beta \sharp}+\mathcal{P}_{\varepsilon}^{\beta \alpha \sharp]}\right]\left(x+(t+\tau)\left(\xi-\xi_{*}\right), \xi_{*}\right) \\
& \quad \times d \tau d \xi_{*} d \xi d x \\
:= & -\Lambda^{\alpha \beta}\left(f_{\varepsilon}(t)\right)+\mathcal{K}_{1}(t)+\mathcal{K}_{2}(t) .
\end{aligned}
$$

Case $1\left(\mathcal{K}_{1}(t)\right)$ : Recall the pointwise estimate

$$
f_{\alpha \varepsilon}^{\sharp}\left(x+(t+\tau)\left(\xi-\xi_{*}\right), \xi_{*}\right) \leq \delta_{\alpha} e^{m_{\alpha} p} e^{-m_{\alpha}\left(\frac{p}{2}\left|x+(t+\tau)\left(\xi-\xi_{*}\right)\right|^{2}+\frac{q}{2}\left|\xi_{*}\right|^{2}\right)}
$$


to find that

$$
\int_{0}^{\infty} \int_{\mathbb{R}^{3}}\left|\xi-\xi_{*}\right| f_{\alpha \varepsilon}^{\sharp}\left(x+(t+\tau)\left(\xi-\xi_{*}\right), \xi_{*}, t\right) d \xi_{*} d \tau \leq 2 C_{1}^{\alpha} \delta_{\alpha} .
$$

We now use the above estimate to see that

$$
\begin{aligned}
\mathcal{K}_{1}(t) & \int_{\mathbb{R}^{6}}\left[\mathcal{Q}_{\varepsilon}^{\alpha \alpha \sharp}\left(f_{\alpha}, f_{\alpha}\right)+\mathcal{Q}_{\varepsilon}^{\alpha \beta \sharp}\left(f_{\alpha}, f_{\beta}\right)(x, \xi, t)+\mathcal{P}_{\varepsilon}^{\alpha \alpha}+\mathcal{P}_{\varepsilon}^{\alpha \beta}\right] \\
& \times\left(\int_{\mathbb{R}^{3} \times \mathbb{R}_{+}}\left|\xi-\xi_{*}\right| f_{\beta \varepsilon}^{\sharp}\left(x+(t+\tau)\left(\xi-\xi_{*}\right), \xi_{*}, t\right) d \tau d \xi_{*}\right) d \xi d x \\
\leq & C \delta_{\beta} \int_{\mathbb{R}^{6}}\left[\left(Q_{+\varepsilon}^{\alpha \alpha \sharp}+Q_{-\varepsilon}^{\alpha \alpha \sharp}\right)\left(f_{\alpha}, f_{\alpha}\right)+\left(Q_{+\varepsilon}^{\alpha \beta \sharp}+Q_{-\varepsilon}^{\alpha \beta \sharp}\right)\left(f_{\alpha}, f_{\beta}\right)\right] d \xi d x \\
+ & C \delta_{\beta} \int_{\mathbb{R}^{6}}\left|\mathcal{P}_{\varepsilon}^{\alpha \alpha \sharp}+\mathcal{P}_{\varepsilon}^{\alpha \beta \sharp}\right| d \xi d x \\
\leq & C \delta_{\beta}\left[\Lambda^{\alpha \alpha}\left(f_{\varepsilon}(t)\right)+\Lambda^{\alpha \beta}\left(f_{\varepsilon}(t)\right)\right]+C \delta_{\beta}\left(\int_{\mathbb{R}^{3} \times \mathbb{R}^{3}}\left|\mathcal{P}_{\varepsilon}^{\alpha \alpha \sharp}+\mathcal{P}_{\varepsilon}^{\alpha \beta \sharp}\right| d x d \xi\right),
\end{aligned}
$$

where we use Lemma 5.2.

Case $2\left(\mathcal{K}_{2}(t)\right)$ : Similar to Case 1 , we have

$$
\mathcal{K}_{2}(t) \leq C \delta_{\alpha}\left[\Lambda^{\beta \beta}\left(f_{\varepsilon}(t)\right)+\Lambda^{\beta \alpha}\left(f_{\varepsilon}(t)\right)\right]+C \delta_{\alpha}\left(\int_{\mathbb{R}^{6}}\left|\mathcal{P}_{\varepsilon}^{\beta \beta \sharp}+\mathcal{P}_{\varepsilon}^{\beta \alpha \sharp}\right| d x d \xi\right) .
$$

We now have the bound of time-derivative of $D^{\alpha \beta}\left(f_{\varepsilon}(t)\right)$.

$$
\begin{aligned}
\frac{d}{d t} D^{\alpha \beta}\left(f_{\varepsilon}(t)\right) \leq & -\Lambda^{\alpha \beta}\left(f_{\varepsilon}(t)\right) \\
& +C \delta_{\beta}\left[\Lambda^{\alpha \alpha}\left(f_{\varepsilon}(t)\right)+\Lambda^{\alpha \beta}\left(f_{\varepsilon}(t)\right)\right]+C \delta_{\alpha}\left[\Lambda^{\beta \beta}\left(f_{\varepsilon}(t)\right)+\Lambda^{\alpha \beta}\left(f_{\varepsilon}(t)\right)\right] \\
& +C\left(\delta_{\alpha}+\delta_{\beta}\right) \sum_{\alpha \beta \in\{A, B\}} \int_{\mathbb{R}^{6}}\left|\mathcal{P}_{ \pm \varepsilon}^{\alpha \beta \sharp}\right| d x d \xi .
\end{aligned}
$$

We now sum up over $\alpha, \beta \in\{A, B\}$ to find

$$
\begin{aligned}
\frac{d}{d t} D\left(f_{\varepsilon}(t)\right) & =\sum_{\alpha, \beta \in\{A, B\}} \frac{d}{d t} D^{\alpha \beta}\left(f_{\varepsilon}(t)\right) \\
& \leq\left[-1+C\left(\delta_{A}+\delta_{B}\right)\right] \Lambda\left(f_{\varepsilon}(t)\right)+C\left(\delta_{\alpha}+\delta_{\beta}\right) \int_{\mathbb{R}^{6}} \mathcal{P}(x, \xi, \varepsilon) d x d \xi \\
& \leq-C_{1} \Lambda\left(f_{\varepsilon}(t)\right)+C \int_{\mathbb{R}^{6}} \mathcal{P}(x, \xi, \varepsilon) d x d \xi,
\end{aligned}
$$

where we take $\delta_{\alpha}, \delta_{\beta}$ small enough so that $C_{1}$ is positive. We integrate the above inequality from $s=0$ to $s=t$ to see that

$$
\mathcal{D}\left(f_{\varepsilon}(t)\right)+C_{1} \int_{0}^{t} \Lambda\left(f_{\varepsilon}(s)\right) d s \leq \mathcal{D}\left(f_{\varepsilon}(0)\right)+C \int_{0}^{t} \int_{\mathbb{R}^{6}} \mathcal{P}(x, \xi, \varepsilon) d x d \xi d s .
$$

Let $\varepsilon \rightarrow 0$. The phase space integral of perturbation goes to zero as $\varepsilon \rightarrow 0$ from Lemma 5.1 and then we obtain

$$
\mathcal{D}(f(t))+C_{1} \int_{0}^{t} \Lambda(f(s)) d s \leq \mathcal{D}\left(f_{0}\right), \quad t \geq 0 .
$$


The proof of Theorem 2.1 (scattering estimate). We set the time-asymptotic state $F_{\alpha+}$ :

$$
F_{\alpha+}(x, \xi):=F_{\alpha 0}(x, \xi)+\int_{0}^{\infty}\left[Q^{\alpha \alpha \sharp}\left(F_{\alpha}, F_{\alpha}\right)+Q^{\alpha \beta \sharp}\left(F_{\alpha}, F_{\beta}\right)\right](x, \xi, s) d s .
$$

Then it is easy to see that $F_{\alpha+} \in L^{1}\left(\mathbb{R}^{6}\right)$ and the weighted $L_{r}^{1}$ difference is given by

$$
\begin{aligned}
\sum_{\alpha \in\{A, B\}} & \left\|F_{\alpha}(x+t \xi, \xi, t)-F_{\alpha+}(x, \xi)\right\|_{L_{\alpha, r}^{1}} \\
\leq & \sum_{\alpha \in\{A, B\}}\left\|f_{\alpha}(x+t \xi, \xi, t)-f_{\alpha+}(x, \xi)\right\|_{L^{1}} \\
\leq & \int_{t}^{\infty} \int_{\mathbb{R}^{6}} \sum_{\alpha, \beta \in\{A, B\}}\left|\mathcal{Q}^{\alpha \beta \sharp}\left(f_{\alpha}, f_{\beta}\right)\right|(x, \xi, s) d \xi d x d s .
\end{aligned}
$$

On the other hand, it follows from Proposition 5.1 that we have

$$
\begin{aligned}
& \int_{0}^{\infty} \int_{\mathbb{R}^{6}} \sum_{\alpha, \beta \in\{A, B\}}\left|\mathcal{Q}^{\alpha \sharp \sharp}\left(f_{\alpha}, f_{\beta}\right)\right|(x, \xi) d \xi d x d t \\
& \quad \leq C \sum_{\alpha, \beta \in\{A, B\}} \int_{0}^{\infty} \int_{\mathbb{R}^{6}}\left(Q_{+}^{\alpha \beta \sharp}+Q_{-}^{\alpha \beta \sharp}\right)\left(f_{\alpha}, f_{\beta}\right)(x, \xi) d \xi d x d t \\
& \quad \leq C \sum_{\alpha, \beta \in\{A, B\}} \int_{0}^{\infty} \Lambda^{\alpha \beta \sharp}(f(t)) d t \\
& \quad=C \int_{0}^{\infty} \Lambda(f(t)) d t \leq C \mathcal{D}\left(f_{0}\right),
\end{aligned}
$$

which gives us

$$
\sum_{\alpha, \beta \in\{A, B\}} \int_{t}^{\infty} \int_{\mathbb{R}^{6}}\left|\mathcal{Q}^{\alpha \beta \sharp}\left(f_{\alpha}, f_{\beta}\right)\right|(x, \xi) d \xi d x d s \rightarrow 0, \quad \text { as } t \rightarrow \infty .
$$

This completes the proof.

6. Uniform weighted $L^{1}$-stability estimate. In this section, we study the uniform weighted $L^{1}$-stability estimates with the weight $\varphi_{\alpha, r}$ in the velocity space to establish the uniform $L^{1}$-stability estimate. We use the nonlinear functional approach as in [17, 19]. Similar to the previous section, we define nonlinear collision potential functionals $\mathcal{D}_{d \varepsilon}^{\alpha \beta}(t)$ and collision production rates $\Lambda_{d \varepsilon}^{\alpha \beta}(t)$ :

$$
\begin{aligned}
\mathcal{D}_{d \varepsilon}^{\alpha \beta}(t):=\int_{\mathbb{R}^{6}}\left|f_{\alpha \varepsilon}-\bar{f}_{\alpha \varepsilon}\right|^{\sharp}(x, \xi)\left[\int_{0}^{\infty} \int_{\mathbb{R}^{3}}\left|\xi-\xi_{*}\right|\right. \\
\left.\times\left(f_{\beta \varepsilon}^{\sharp}+\bar{f}_{\beta \varepsilon}^{\sharp}\right)\left(x+(t+\tau)\left(\xi-\xi_{*}\right), \xi_{*}\right) d \xi_{*} d \tau\right] d \xi d x, \\
\Lambda_{d \varepsilon}^{\alpha \beta}(t):=\int_{\mathbb{R}^{9}}\left|\xi-\xi_{*}\right|\left|f_{\alpha \varepsilon}-\bar{f}_{\alpha \varepsilon}\right|^{\sharp}(x, \xi)\left(f_{\beta \varepsilon}^{\sharp}+\bar{f}_{\beta \varepsilon}^{\sharp}\right)\left(x+t\left(\xi-\xi_{*}\right), \xi_{*}\right) d \xi_{*} d \xi d x, \\
\mathcal{D}_{d \varepsilon}(t):=\sum_{\alpha, \beta \in\{A, B\}} \mathcal{D}_{d \varepsilon}^{\alpha \beta}(t), \quad \Lambda_{d \varepsilon}(t):=\sum_{\alpha, \beta \in\{A, B\}} \Lambda_{d \varepsilon}^{\alpha \beta}(t),
\end{aligned}
$$


and we also define the nonlinear functional which is equivalent to $L_{\varphi}^{1}$-distance:

$$
\mathcal{H}_{\varepsilon}(t):=\sum_{\alpha \in\{A, B\}}\left\|f_{\alpha \varepsilon}(t)-\bar{f}_{\alpha \varepsilon}(t)\right\|_{L^{1}}+K \mathcal{D}_{d \varepsilon}(t), \quad \mathcal{H}(t):=\lim _{\varepsilon \rightarrow 0+} \mathcal{H}_{\varepsilon}(t),
$$

where $K$ is a positive constant to be determined later.

The equivalence between $\mathcal{H}_{\varepsilon}(t)$ and $L_{\varphi}^{1}$-distance can be checked as follows.

$$
\begin{aligned}
& \left\|f_{\alpha \varepsilon}(t)-\bar{f}_{\alpha \varepsilon}(t)\right\|_{L^{1}} \\
& =\int_{\mathbb{R}^{6}}\left|f_{\alpha \varepsilon}-\bar{f}_{\alpha \varepsilon}\right|^{\sharp}(x, \xi) \\
& \quad \times\left[1+K \int_{0}^{\infty} \int_{\mathbb{R}^{3}}\left|\xi-\xi_{*}\right| \sum_{\eta \in\{\alpha, \beta\}}\left(f_{\eta \varepsilon}^{\sharp}+\bar{f}_{\eta \varepsilon}^{\sharp}\right)\left(x+(t+\tau)\left(\xi-\xi_{*}\right), \xi_{*}\right) d \xi_{*} d \tau\right] d \xi d x \\
& \quad \leq\left(1+2 K\left(C_{1}^{\alpha}+C_{1}^{\beta}\right)\right)\left\|f_{\alpha \varepsilon}(t)-\bar{f}_{\alpha \varepsilon}(t)\right\|_{L^{1}} .
\end{aligned}
$$

We next derive the differential inequality for $\left|f_{\alpha}-\bar{f}_{\alpha}\right|$. Recall that $f_{\alpha \varepsilon}$ and $\bar{f}_{\alpha \varepsilon}$ satisfy

$$
\begin{aligned}
& \partial_{t} f_{\alpha \varepsilon}+\xi \cdot \nabla_{x} f_{\alpha \varepsilon}=\mathcal{Q}^{\alpha \alpha}\left(f_{\alpha \varepsilon}, f_{\alpha \varepsilon}\right)+\mathcal{Q}^{\alpha \beta}\left(f_{\alpha \varepsilon}, f_{\beta \varepsilon}\right)+\mathcal{P}_{\varepsilon}^{\alpha \alpha}+\mathcal{P}_{\varepsilon}^{\alpha \beta}, \\
& \partial_{t} \bar{f}_{\alpha \varepsilon}+\xi \cdot \nabla_{x} \bar{f}_{\alpha \varepsilon}=\mathcal{Q}^{\alpha \alpha}\left(\bar{f}_{\alpha \varepsilon}, \bar{f}_{\alpha \varepsilon}\right)+\mathcal{Q}^{\alpha \beta}\left(\bar{f}_{\alpha \varepsilon}, \bar{f}_{\beta \varepsilon}\right)+\overline{\mathcal{P}}_{\varepsilon}^{\alpha \alpha}+\overline{\mathcal{P}}_{\varepsilon}^{\alpha \beta} .
\end{aligned}
$$

Then we subtract the equations and multiply the resulting equation by $\operatorname{sgn}\left(f_{\alpha \varepsilon}-\bar{f}_{\alpha \varepsilon}\right)$ to find

$$
\partial_{t}\left|f_{\alpha \varepsilon}-\bar{f}_{\alpha \varepsilon}\right|+\xi \cdot \nabla_{x}\left|f_{\alpha \varepsilon}-\bar{f}_{\alpha \varepsilon}\right| \leq \mathcal{R}_{\varepsilon}^{\alpha \alpha}+\mathcal{R}_{\varepsilon}^{\alpha \beta}+\mathcal{P}_{d \varepsilon}^{\alpha \alpha}+\mathcal{P}_{d \varepsilon}^{\alpha \beta}
$$

where

$$
\begin{array}{ll}
\mathcal{R}_{\varepsilon}^{\alpha \beta}:=\frac{1}{2} \int_{\mathbb{R}^{3} \times \mathbf{S}^{2}}\left|\left(\xi-\xi_{*}\right) \cdot \omega\right|\left(\frac{\left|f_{\alpha \varepsilon}^{\prime}-\bar{f}_{\alpha \varepsilon}^{\prime}\right|\left(f_{\beta * \varepsilon}^{\prime}+\bar{f}_{\beta * \varepsilon}^{\prime}\right)}{e^{\prime} J^{\prime}}+\frac{\left|f_{\beta * \varepsilon}^{\prime}-\bar{f}_{\beta * \varepsilon}^{\prime}\right|\left(f_{\alpha \varepsilon}^{\prime}+\bar{f}_{\alpha \varepsilon}^{\prime}\right)}{e^{\prime} J^{\prime}}\right. & \left.+\left|f_{\alpha \varepsilon}-\bar{f}_{\alpha \varepsilon}\right|\left(f_{\beta * \varepsilon}+\bar{f}_{\beta * \varepsilon}\right)+\left|f_{\beta * \varepsilon}-\bar{f}_{\beta * \varepsilon}\right|\left(f_{\alpha \varepsilon}+\bar{f}_{\alpha \varepsilon}\right)\right) d \omega d \xi_{*}, \\
\mathcal{P}_{d \varepsilon}^{\alpha \beta}:=\left|\mathcal{P}_{\varepsilon}^{\alpha \beta}-\overline{\mathcal{P}}_{\varepsilon}^{\alpha \beta}\right|, & \mathcal{P}_{d}(x, \xi, \varepsilon):=\sum_{\alpha, \beta \in\{A, B\}} \mathcal{P}_{d \varepsilon}^{\alpha \beta} .
\end{array}
$$

Lemma 6.1. Let $f_{\alpha}$ and $\bar{f}_{\alpha}$ be the re-scaled function of mild solutions $F_{\alpha}$ and $\bar{F}_{\alpha}$ respectively. Then we have

$$
\int_{\mathbb{R}^{6}} \mathcal{R}_{\varepsilon}^{\alpha \beta \sharp} d \xi d x \leq 8 \pi \Lambda_{d \varepsilon}^{\alpha \beta}(t) .
$$

Proof. We use the change of variables $\left(\xi^{\prime}, \xi_{*}^{\prime}\right) \leftrightarrow\left(\xi, \xi_{*}\right)$ and the Fubini theorem to have

$$
\begin{aligned}
& \int_{\mathbb{R}^{6}} \mathcal{R}_{\varepsilon}^{\alpha \beta \sharp} d \xi d x \\
& =\frac{1}{2} \int_{\mathbb{R}^{9} \times \mathbf{S}^{2}}\left|\left(\xi-\xi_{*}\right) \cdot \omega\right|\left(\frac{\left|f_{\alpha \varepsilon}^{\prime}-\bar{f}_{\alpha \varepsilon}^{\prime}\right|\left(f_{\beta * \varepsilon}^{\prime}+\bar{f}_{\beta * \varepsilon}^{\prime}\right)}{e^{\prime} J^{\prime}}+\frac{\left|f_{\beta * \varepsilon}^{\prime}-\bar{f}_{\beta * \varepsilon}^{\prime}\right|\left(f_{\alpha \varepsilon}^{\prime}+\bar{f}_{\alpha \varepsilon}^{\prime}\right)}{e^{\prime} J^{\prime}}\right. \\
& \left.\quad+\left|f_{\alpha \varepsilon}-\bar{f}_{\alpha \varepsilon}\right|\left(f_{\beta * \varepsilon}+\bar{f}_{\beta * \varepsilon}\right)+\left|f_{\beta * \varepsilon}-\bar{f}_{\beta * \varepsilon}\right|\left(f_{\alpha \varepsilon}+\bar{f}_{\alpha \varepsilon}\right)\right) d \omega d \xi_{*} d \xi d x \\
& =2 \int_{\mathbb{R}^{9} \times \mathbf{S}^{2}}\left|\left(\xi-\xi_{*}\right) \cdot \omega\right|\left|f_{\alpha \varepsilon}-\bar{f}_{\alpha \varepsilon}\right|\left(f_{\beta * \varepsilon}+\bar{f}_{\beta * \varepsilon}\right) d \omega d \xi_{*} d \xi d x \\
& =8 \pi \int_{\mathbb{R}^{9}}\left|\xi-\xi_{*}\right|\left|f_{\alpha \varepsilon}-\bar{f}_{\alpha \varepsilon}\right|\left(f_{\beta * \varepsilon}+\bar{f}_{\beta * \varepsilon}\right) d \xi_{*} d \xi d x
\end{aligned}
$$

Hence we get the desired result. 
Before we derive the differential inequality of $\mathcal{H}_{\varepsilon}(t)$, we set the following auxiliary functionals:

$$
\begin{aligned}
\mathcal{E}_{\varepsilon}^{\alpha \beta}(x, \xi, t) & \\
& :=\int_{0}^{\infty} \int_{\mathbb{R}^{3}}\left|\xi-\xi_{*}\right| \mathcal{Q}_{+}^{\alpha \beta \sharp}\left(f_{\alpha \varepsilon}, f_{\beta \varepsilon}\right)\left(x+(t+\tau)\left(\xi-\xi_{*}\right), \xi_{*}\right) d \xi_{*} d \tau, \\
\mathcal{G}_{\varepsilon}^{\alpha \beta}(x, \xi, t) & :=\int_{0}^{\infty} \int_{\mathbb{R}^{3}}\left|\xi-\xi_{*}\right| \mathcal{P}_{\varepsilon}^{\alpha \beta \sharp}\left(x+(t+\tau)\left(\xi-\xi_{*}\right), \xi_{*}\right) d \xi_{*} d \tau, \\
\mathcal{E}_{\varepsilon}(t) & :=\sum_{\alpha, \beta \in\{A, B\}} \mathcal{E}_{\varepsilon}^{\alpha \beta}(t), \quad \mathcal{G}_{\varepsilon}(t):=\sum_{\alpha, \beta \in\{A, B\}} \mathcal{G}_{\varepsilon}^{\alpha \beta}(t) .
\end{aligned}
$$

Next lemma shows that these auxiliary functionals have time-decay estimates.

Lemma 6.2. Let $f_{\alpha \varepsilon}=F_{\alpha \varepsilon} \varphi_{\alpha, r}$ be the re-scaled function of the mild solution $F_{\alpha}$ to (5.3). Then we have

$$
\left(\mathcal{E}_{\varepsilon}+\mathcal{G}_{\varepsilon}\right)(x, \xi, t) \leq \frac{C}{(t+1)^{4}}, \quad x, \xi \in \mathbb{R}^{3}, t \geq 0 .
$$

Proof. (i) The estimate of $\mathcal{E}_{\mathcal{\varepsilon}}^{\alpha \beta}(t)$ can be calculated similarly to Lemma 4.2 and 4.3 . For fixed $x, \xi \in \mathbb{R}^{3}$, we have the bound of a collision operator:

$$
\left.\mathcal{Q}_{+}^{\alpha \beta \sharp}\left(f_{\alpha \varepsilon}, f_{\beta \varepsilon}\right)\left(x+(t+\tau)\left(\xi-\xi_{*}\right), \xi_{*}\right) \leq \frac{C_{1}^{\alpha \beta} \delta_{\alpha} \delta_{\beta}}{(t+1)^{4}} e^{-m_{\alpha}\left(\frac{p}{3}\left|x+(t+\tau)\left(\xi-\xi_{*}\right)\right|^{2}+\frac{q}{3}\left|\xi_{*}\right|^{2}\right.}\right) .
$$

We calculate the integral as we did in the proof of Lemma 3.1 to get

$$
\begin{aligned}
\mathcal{E}_{\varepsilon}^{\alpha \beta}(x, & \xi, t) \\
& \leq \frac{C_{1}^{\alpha \beta} \delta_{\alpha} \delta_{\beta}}{(t+1)^{4}} \int_{\mathbb{R}^{3}}\left|\xi-\xi_{*}\right| e^{-m_{\alpha} \frac{q}{3}\left|\xi_{*}\right|^{2}}\left(\int_{0}^{\infty} e^{-m_{\alpha} \frac{p}{3}\left|x+(t+\tau)\left(\xi-\xi_{*}\right)\right|^{2}} d \tau\right) d \xi_{*} \\
& \leq \frac{C_{1}^{\alpha \beta} \delta_{\alpha} \delta_{\beta}}{(t+1)^{4}} \sqrt{\frac{3 \pi}{p}} \int_{\mathbb{R}^{3}} e^{-m_{\alpha} \frac{q}{3}\left|\xi_{*}\right|^{2}} d \xi_{*} \\
& \leq \frac{C}{(t+1)^{4}} .
\end{aligned}
$$

(ii) The estimate for $\mathcal{G}_{\varepsilon}^{\alpha \beta}(t)$ : Note that

$$
\begin{aligned}
\left|\mathcal{P}_{ \pm}^{\alpha \beta}(x, \xi, t)\right| & \leq\left|\mathcal{Q}_{ \pm \varepsilon}^{\alpha \beta \sharp}\left(f_{\alpha}, f_{\beta}\right)(x, \xi, t)\right|+\left|\mathcal{Q}_{ \pm}^{\alpha \beta \sharp}\left(f_{\alpha \varepsilon}, f_{\beta \varepsilon}\right)(x, \xi, t)\right| \\
& \leq C C_{1}^{\alpha \beta} \delta_{\alpha} \delta_{\beta}\left(\frac{e^{-m_{\alpha}\left(\frac{p}{2}|x|^{2}+\frac{q}{3}|\xi|^{2}\right)}}{(t+1)^{4}}\right)_{\varepsilon}+C C_{1}^{\alpha \beta} \delta_{\alpha} \delta_{\beta} \frac{e^{-m_{\alpha}\left(\frac{p}{3}|x|^{2}+\frac{q}{3}|\xi|^{2}\right)}}{(t+1)^{4}} \\
& \leq C C_{1}^{\alpha \beta} \delta_{\alpha} \delta_{\beta} \frac{e^{-m_{\alpha}\left(\frac{p}{3}|x|^{2}+\frac{q}{3}|\xi|^{2}\right)}}{(t+1)^{4}} .
\end{aligned}
$$

Then we can do the same analysis as in (1) to get the time-decay estimate.

Proposition 6.1. Let $f_{\alpha}=F_{\alpha} \varphi_{\alpha, r}$ be the re-scaled function of the mild solution $F_{\alpha}$ constructed in Section 3. Then we have

$$
\begin{aligned}
& \text { (i) } \frac{d}{d t} \sum_{\alpha \in\{A, B\}}\left\|f_{\alpha \varepsilon}(t)-\bar{f}_{\alpha \varepsilon}(t)\right\|_{L^{1}} \leq 8 \pi \Lambda_{d \varepsilon}(t)+C \int_{\mathbb{R}^{3} \times \mathbb{R}^{3}} \mathcal{P}_{d}^{\sharp}(x, \xi, \varepsilon) d \xi d x . \\
& \text { (ii) } \frac{d}{d t} \mathcal{D}_{d \varepsilon}(t) \leq-C_{1} \Lambda_{d \varepsilon}(t)+\frac{C}{(t+1)^{4}} \sum_{\alpha \in\{A, B\}}\left\|f_{\alpha \varepsilon}(t)-\bar{f}_{\alpha \varepsilon}(t)\right\|_{L^{1}}
\end{aligned}
$$




$$
+C \int_{\mathbb{R}^{6}} \mathcal{P}_{d}^{\sharp}(x, \xi, \varepsilon) d \xi d x .
$$

Proof. (i) Recall (6.1) to see

$$
\partial_{t}\left|f_{\alpha \varepsilon}-\bar{f}_{\alpha \varepsilon}\right|^{\sharp} \leq \mathcal{R}_{\varepsilon}^{\alpha \alpha \sharp}+\mathcal{R}_{\varepsilon}^{\alpha \beta \sharp}+\mathcal{P}_{d \varepsilon}^{\alpha \alpha \sharp}+\mathcal{P}_{d \varepsilon}^{\alpha \beta \sharp},
$$

and we integrate (6.1) over $\mathbb{R}^{6}$ to find

$$
\begin{aligned}
& \frac{d}{d t} \sum_{\alpha \in\{A, B\}}\left\|f_{\alpha \varepsilon}(t)-\bar{f}_{\alpha \varepsilon}(t)\right\|_{L^{1}} \\
& \quad \leq \sum_{\alpha, \beta \in\{A, B\}}\left(\int_{\mathbb{R}^{6}} \mathcal{R}_{\varepsilon}^{\alpha \beta \sharp} d \xi d x+\int_{\mathbb{R}^{6}} \mathcal{P}_{d \varepsilon}^{\alpha \beta \sharp} d \xi d x\right) \\
& \quad \leq 8 \pi \Lambda_{d \varepsilon}(t)+\sum_{\alpha, \beta \in\{A, B\}} \int_{\mathbb{R}^{6}} \mathcal{P}_{d \varepsilon}^{\alpha \beta \sharp} d \xi d x . \\
& \quad \leq 8 \pi \Lambda_{d \varepsilon}(t)+\int_{\mathbb{R}^{6}} \mathcal{P}_{d}^{\sharp}(x, \xi, \varepsilon) d \xi d x .
\end{aligned}
$$

(ii) To calculate the time-derivative of $\mathcal{D}_{d \varepsilon}^{\alpha \beta}(t)$, we estimate that of the integrand. Relation (5.7) and (6.1) give

$$
\begin{aligned}
& \partial_{t}\left(\left|\xi-\xi_{*}\right|\left|f_{\alpha \varepsilon}-\bar{f}_{\alpha \varepsilon}\right|^{\sharp}(x, \xi)\left(f_{\beta \varepsilon}^{\sharp}+\bar{f}_{\beta \varepsilon}^{\sharp}\right)\left(x+(t+\tau)\left(\xi-\xi_{*}\right), \xi_{*}\right)\right) \\
& \leq \partial_{\tau}\left(\left|\xi-\xi_{*}\right|\left|f_{\alpha \varepsilon}-\bar{f}_{\alpha \varepsilon}\right|^{\sharp}(x, \xi)\left(f_{\varepsilon}^{\beta \sharp}+\bar{f}_{\varepsilon}^{\beta \sharp}\right)\left(x+(t+\tau)\left(\xi-\xi_{*}\right), \xi_{*}\right)\right) \\
& +\left|\xi-\xi_{*}\right|\left(\mathcal{R}_{\varepsilon}^{\alpha \alpha \sharp}+\mathcal{R}_{\varepsilon}^{\alpha \beta \sharp}+\mathcal{P}_{d \varepsilon}^{\alpha \alpha \sharp}+\mathcal{P}_{d \varepsilon}^{\alpha \beta \sharp}\right)(x, \xi)\left(f_{\varepsilon}^{\beta \sharp}+\bar{f}_{\varepsilon}^{\beta \sharp}\right)\left(x+(t+\tau)\left(\xi-\xi_{*}\right), \xi_{*}\right) \\
& +\left|\xi-\xi_{*}\right|\left|f_{\alpha \varepsilon}-\bar{f}_{\alpha \varepsilon}\right|^{\sharp}(x, \xi)\left(\mathcal{Q}^{\beta \beta \sharp}\left(f_{\beta \varepsilon}, f_{\beta \varepsilon}\right)+\mathcal{Q}^{\beta \alpha \sharp}\left(f_{\beta \varepsilon}, f_{\alpha \varepsilon}\right)\right. \\
& \left.+\mathcal{Q}^{\beta \beta \sharp}\left(\bar{f}_{\beta \varepsilon}, \bar{f}_{\beta \varepsilon}\right)+\mathcal{Q}^{\beta \alpha \sharp}\left(\bar{f}_{\beta \varepsilon}, \bar{f}_{\alpha \varepsilon}\right)\right)\left(x+(t+\tau)\left(\xi-\xi_{*}\right), \xi_{*}\right) \\
& +\left|\xi-\xi_{*}\right|\left|f_{\alpha \varepsilon}-\bar{f}_{\alpha \varepsilon}\right|^{\sharp}(x, \xi)\left(\mathcal{P}_{\varepsilon}^{\beta \beta \sharp}+\mathcal{P}_{\varepsilon}^{\beta \alpha \sharp}+\overline{\mathcal{P}}_{\varepsilon}^{\beta \beta \sharp}+\overline{\mathcal{P}}_{\varepsilon}^{\beta \alpha \sharp}\right)\left(x+(t+\tau)\left(\xi-\xi_{*}\right), \xi_{*}\right) .
\end{aligned}
$$

We integrate the above inequality over $\mathbb{R}^{9} \times \mathbb{R}_{+}$with respect to $\left(x, \xi, \xi_{*}, \tau\right)$ to get

$$
\begin{aligned}
& \frac{d \mathcal{D}_{d \varepsilon}^{\alpha \beta}(t)}{d t} \\
& \leq-\int_{\mathbb{R}^{9}}\left|\xi-\xi_{*}\right|\left|f_{\alpha \varepsilon}-\bar{f}_{\alpha \varepsilon}\right|^{\sharp}(x, \xi)\left(f_{\beta \varepsilon}^{\sharp}+\bar{f}_{\beta \varepsilon}^{\sharp}\right)\left(x+t\left(\xi-\xi_{*}\right), \xi_{*}\right) d \xi d \xi_{*} d x \\
& +\int_{0}^{\infty} \int_{\mathbb{R}^{9}}\left|\xi-\xi_{*}\right|\left(\mathcal{R}_{\varepsilon}^{\alpha \alpha \sharp}+\mathcal{R}_{\varepsilon}^{\alpha \beta \sharp}+\mathcal{P}_{d \varepsilon}^{\alpha \alpha \sharp}+\mathcal{P}_{d \varepsilon}^{\alpha \beta \sharp}\right)(x, \xi) \\
& \times\left(f_{\beta \varepsilon}^{\sharp}+\bar{f}_{\beta \varepsilon}^{\sharp}\right)\left(x+(t+\tau)\left(\xi-\xi_{*}\right), \xi_{*}\right) d \xi_{*} d \xi d x d \tau \\
& +\int_{0}^{\infty} \int_{\mathbb{R}^{9}}\left|\xi-\xi_{*}\right|\left(\mathcal{Q}^{\beta \beta \sharp}\left(f_{\beta \varepsilon}, f_{\beta \varepsilon}\right)+\mathcal{Q}^{\beta \alpha \sharp}\left(f_{\beta \varepsilon}, f_{\alpha \varepsilon}\right)\right. \\
& \left.+\mathcal{Q}^{\beta \beta \sharp}\left(\bar{f}_{\beta \varepsilon}, \bar{f}_{\beta \varepsilon}\right)+\mathcal{Q}^{\beta \alpha \sharp}\left(\bar{f}_{\beta \varepsilon}, \bar{f}_{\alpha \varepsilon}\right)\right)\left(\left(x+(t+\tau)\left(\xi-\xi_{*}\right), \xi_{*}\right), \xi_{*}, t\right) \\
& \times\left|f_{\alpha \varepsilon}-\bar{f}_{\alpha \varepsilon}\right|^{\sharp}(x, \xi) d \xi_{*} d \xi d x d \tau \\
& +\int_{0}^{\infty} \int_{\mathbb{R}^{9}}\left|\xi-\xi_{*}\right|\left|f_{\alpha \varepsilon}-\bar{f}_{\alpha \varepsilon}\right|^{\sharp}(x, \xi) \\
& \times\left(\mathcal{P}_{\varepsilon}^{\beta \beta \sharp}+\mathcal{P}_{\varepsilon}^{\beta \alpha \sharp}+\mathcal{P}_{\varepsilon}^{\beta \beta \sharp}+\mathcal{P}_{\varepsilon}^{\beta \alpha \sharp}\right)\left(x+(t+\tau)\left(\xi-\xi_{*}\right), \xi_{*}\right) d \xi_{*} d \xi d x d \tau \\
& :=-\Lambda_{d \varepsilon}^{\alpha \beta}(t)+\mathcal{N}_{1}(t)+\mathcal{N}_{2}(t)+\mathcal{N}_{3}(t) \text {. }
\end{aligned}
$$


Next we estimate $\mathcal{N}_{i}$ as follows.

Case $1\left(\mathcal{N}_{1}\right)$ : We use Lemma 4.2 and Lemma 6.1 to see

$$
\begin{aligned}
\mathcal{N}_{1}(t)= & \int_{\mathbb{R}^{6}}\left(\mathcal{R}_{\varepsilon}^{\alpha \alpha \sharp}+\mathcal{R}_{\varepsilon}^{\alpha \beta \sharp}+\mathcal{P}_{d \varepsilon}^{\alpha \alpha \sharp}+\mathcal{P}_{d \varepsilon}^{\alpha \beta \sharp}\right)(x, \xi) \\
& \times\left[\int_{0}^{\infty} \int_{\mathbb{R}^{3}}\left|\xi-\xi_{*}\right|\left(f_{\beta \varepsilon}^{\sharp}+\bar{f}_{\beta \varepsilon}^{\sharp}\right)\left(x+(t+\tau)\left(\xi-\xi_{*}\right), \xi_{*}\right) d \xi_{*} d \tau\right] d \xi d x \\
\leq & 8 \pi \delta_{\beta} C_{1}^{\beta}\left(\Lambda_{\varepsilon}^{\alpha \alpha}(t)+\Lambda_{\varepsilon}^{\alpha \beta}(t)\right)+C \int_{\mathbb{R}^{6}}\left(\mathcal{P}_{d \varepsilon}^{\alpha \alpha \sharp}+\mathcal{P}_{d \varepsilon}^{\alpha \beta \sharp}\right) d \xi d x \\
\leq & 8 \pi\left(\delta_{\alpha}+\delta_{\beta}\right)\left(C_{1}^{\alpha}+C_{1}^{\beta}\right) \Lambda_{d \varepsilon}(t)+C \sum_{\alpha, \beta \in\{A, B\}} \int_{\mathbb{R}^{6}} \mathcal{P}_{d \varepsilon}^{\alpha \beta \sharp} d \xi d x \\
= & 8 \pi\left(\delta_{\alpha}+\delta_{\beta}\right)\left(C_{1}^{\alpha}+C_{1}^{\beta}\right) \Lambda_{d \varepsilon}(t)+C \int_{\mathbb{R}^{6}} \mathcal{P}_{d}^{\sharp}(x, \xi, \varepsilon) d \xi d x .
\end{aligned}
$$

Case $2\left(\mathcal{N}_{2}\right)$ : By Lemma 6.2, we have

$$
\begin{aligned}
& \mathcal{N}_{2}(t) \\
& \leq \int_{\mathbb{R}^{6}}\left|f_{\alpha \varepsilon}-\bar{f}_{\alpha \varepsilon}\right|^{\sharp}(x, \xi)\left[\int _ { 0 } ^ { \infty } \int _ { \mathbb { R } ^ { 3 } } | \xi - \xi _ { * } | \left(\mathcal{Q}_{+}^{\beta \beta \sharp}\left(f_{\beta \varepsilon}, f_{\beta \varepsilon}\right)+\mathcal{Q}_{+}^{\beta \alpha \sharp}\left(f_{\beta \varepsilon}, f_{\alpha \varepsilon}\right)\right.\right. \\
& \left.\left.+\mathcal{Q}_{+}^{\beta \beta \sharp}\left(\bar{f}_{\beta \varepsilon}, \bar{f}_{\beta \varepsilon}\right)+\mathcal{Q}_{+}^{\beta \alpha \sharp}\left(\bar{f}_{\beta \varepsilon}, \bar{f}_{\alpha \varepsilon}\right)\right)\left(x+(t+\tau)\left(\xi-\xi_{*}\right), \xi_{*}\right) d \xi_{*} d \tau\right] d \xi d x \\
& \leq\left(\mathcal{E}_{\varepsilon}^{\alpha \alpha}(t)+\mathcal{E}_{\varepsilon}^{\alpha \beta}(t)+\overline{\mathcal{E}}_{\varepsilon}^{\beta \beta}(t)+\overline{\mathcal{E}}_{\varepsilon}^{\beta \alpha}(t)\right) \sum_{\alpha \in\{A, B\}}\left\|f_{\alpha \varepsilon}(t)-\bar{f}_{\alpha \varepsilon}(t)\right\|_{L^{1}} \\
& \leq \frac{C}{(t+1)^{4}} \sum_{\alpha \in\{A, B\}}\left\|f_{\alpha \varepsilon}(t)-\bar{f}_{\alpha \varepsilon}(t)\right\|_{L^{1}} .
\end{aligned}
$$

Case $3\left(\mathcal{N}_{3}\right)$ : Similar to Case 2, we have

$$
\begin{aligned}
& \mathcal{N}_{3}(t) \\
& \leq \int_{\mathbb{R}^{6}}\left|f_{\alpha \varepsilon}-\bar{f}_{\alpha \varepsilon}\right|^{\sharp}(x, \xi)\left(\int_{0}^{\infty} \int_{\mathbb{R}^{3}}\left|\xi-\xi_{*}\right|\right. \\
& \left.\times\left(\mathcal{P}_{\varepsilon}^{\alpha \alpha \sharp}+\mathcal{P}_{\varepsilon}^{\alpha \beta \sharp}+\overline{\mathcal{P}}_{\varepsilon}^{\beta \beta \sharp}+\overline{\mathcal{P}}_{\varepsilon}^{\beta \alpha \sharp}\right)\left(x+(t+\tau)\left(\xi-\xi_{*}\right), \xi_{*}\right) d \xi_{*} d \tau\right) d \xi d x \\
& \leq\left(\mathcal{G}_{\varepsilon}^{\alpha \alpha}(t)+\mathcal{G}_{\varepsilon}^{\alpha \beta}(t)+\overline{\mathcal{G}}_{\varepsilon}^{\beta \beta}(t)+\overline{\mathcal{G}}_{\varepsilon}^{\beta \alpha}(t)\right) \sum_{\alpha \in\{A, B\}}\left\|f_{\alpha \varepsilon}(t)-\bar{f}_{\alpha \varepsilon}(t)\right\|_{L^{1}} \\
& \leq \frac{C}{(t+1)^{4}} \sum_{\alpha \in\{A, B\}}\left\|f_{\alpha \varepsilon}(t)-\bar{f}_{\alpha \varepsilon}(t)\right\|_{L^{1}} .
\end{aligned}
$$

We combine Cases $1-3$ to obtain

$$
\begin{aligned}
\frac{d}{d t} \mathcal{D}_{d \varepsilon}(t) & =\sum_{\alpha, \beta \in\{A, B\}} \frac{d}{d t} \mathcal{D}_{d \varepsilon}^{\alpha \beta}(t) \\
& \leq\left[-1+\left(\delta_{\alpha}+\delta_{\beta}\right)\left(C_{1}^{\alpha}+C_{1}^{\beta}\right)\right] \Lambda_{d \varepsilon}(t)+C \int_{\mathbb{R}^{3} \times \mathbb{R}^{3}} \mathcal{P}_{d}^{\sharp}(x, \xi, \varphi) d \xi d x \\
& +\frac{C}{(t+1)^{4}} \sum_{\alpha \in\{A, B\}}\left\|f_{\alpha \varepsilon}(t)-\bar{f}_{\alpha \varepsilon}(t)\right\|_{L^{1}} .
\end{aligned}
$$

The proof of Theorem 2.1 (uniform stability). We take $\delta_{\alpha}$ and $\delta_{\beta}$ small enough so that

$$
-1+\left(\delta_{\alpha}+\delta_{\beta}\right)\left(C_{1}^{\alpha}+C_{1}^{\beta}\right) \leq-C_{2}
$$


for some positive constant $C_{2}$, and we also choose $K$ large enough so that

$$
8 \pi-K C_{2} \leq-C_{3}
$$

for some positive constant $C_{3}$. Then it follows from Proposition 6.1 that

$$
\begin{aligned}
\frac{d}{d t} \mathcal{H}_{\varepsilon}(t) & =\frac{d}{d t} \sum_{\alpha \in\{A, B\}}\left\|f_{\alpha \varepsilon}(t)-\bar{f}_{\alpha \varepsilon}(t)\right\|_{L^{1}}+K \frac{d}{d t} \mathcal{D}_{d \varepsilon}(t) \\
& \leq-C_{3} \Lambda_{d \varepsilon}(t)+\frac{C}{(t+1)^{4}} \sum_{\alpha \in\{A, B\}}\left\|f_{\alpha \varepsilon}(t)-\bar{f}_{\alpha \varepsilon}(t)\right\|_{L^{1}} \\
& +K \int_{\mathbb{R}^{3} \times \mathbb{R}^{3}} \mathcal{P}_{d}^{\sharp}(x, \xi, \varepsilon) d \xi d x \\
& \leq-C_{3} \Lambda_{d \varepsilon}(t)+\frac{C}{(t+1)^{4}} \mathcal{H}_{\varepsilon}(t)+K \int_{\mathbb{R}^{3} \times \mathbb{R}^{3}} \mathcal{P}_{d}^{\sharp}(x, \xi, \varepsilon) d \xi d x .
\end{aligned}
$$

We use Gronwall's Lemma to find

$$
\mathcal{H}_{\varepsilon}(t)+C_{3} \int_{0}^{t} \Lambda_{d \varepsilon}(s) d s \leq \int_{0}^{t} \int_{\mathbb{R}^{3} \times \mathbb{R}^{3}} \mathcal{P}_{d}^{\sharp}(x, \xi, \varepsilon) d \xi d x d s+C_{4} \mathcal{H}_{\varepsilon}(0) .
$$

We let $\varepsilon \rightarrow 0$ and apply Lemma 5.1 to find

$$
\mathcal{H}(t)+C_{3} \int_{0}^{t} \Lambda_{d}(s) d s \leq C_{4} \mathcal{H}(0)
$$

The $L_{r}^{1}$-stability of mild solutions is given by the non-increment of $\mathcal{H}(t)$ and the equivalence between $\mathcal{H}(t)$ and $\|\cdot\|_{L^{1}}$ :

$$
\sum_{\alpha \in\{A, B\}}\left\|f_{\alpha}(t)-\bar{f}_{\alpha}(t)\right\|_{L^{1}} \leq \mathcal{H}(t) \leq C_{4} \mathcal{H}(0) \leq C_{4} C_{0} \sum_{\alpha \in\{A, B\}}\left\|f_{\alpha 0}-\bar{f}_{\alpha 0}\right\|_{L^{1}} .
$$

Recall that $\sum_{\alpha \in\{A, B\}}\left\|F_{\alpha}(t)-\bar{F}_{\alpha}(t)\right\|_{L_{\alpha, r}^{1}}=\sum_{\alpha \in\{A, B\}}\left\|f_{\alpha}(t)-\bar{f}_{\alpha}(t)\right\|_{L^{1}}$ and set

$$
G:=C_{0} C_{4}
$$

to complete the proof.

7. Generalized Bony potential. In this section, we introduce an interaction potential which slightly generalizes the Bony-type potential in [10, 20].

7.1. Construction of potential. Let $\omega: \mathbb{R}^{3} \rightarrow \mathbb{R}$ be a $C^{2}$-convex and radially symmetric function with the special form

$$
\omega(x)=\varphi(|x|),
$$

where $\varphi: \mathbb{R} \rightarrow \mathbb{R}$ is a convex function with the properties

$$
\varphi^{\prime} \geq 0, \quad \varphi^{\prime} \in L^{\infty}(\mathbb{R}) \quad \text { and } \quad \varphi^{\prime \prime} \geq 0, \quad \text { for } \quad x \geq 0 .
$$

Then we use (7.1) and the identity

$$
\partial_{j} \partial_{i} \omega(x)=\varphi^{\prime \prime}(|x|) \frac{x_{i} x_{j}}{|x|^{2}}+\frac{\varphi^{\prime}(|x|)}{|x|}\left(\delta_{i j}-\frac{x_{i} x_{j}}{|x|^{2}}\right)
$$


to see that the Hessian matrix $\nabla^{2} \omega$ is positive definite:

$$
\sum_{1 \leq i, j \leq 3} \partial_{j} \partial_{i} \omega(x) y_{i} y_{j}=\varphi^{\prime \prime}(|x|) \frac{(x \cdot y)^{2}}{|x|^{2}}+\varphi^{\prime}(|x|)\left(|y|^{2}-\frac{(x \cdot y)^{2}}{|x|^{2}}\right) \geq 0 .
$$

Let $F_{A \varepsilon}$ and $F_{B \varepsilon}$ be the mild solutions to the Boltzmann system (1.1), which was obtained from the mollified approximate solutions $F_{A \varepsilon}$ and $F_{B \varepsilon}$ to the system (4.1). Below we present the construction of the potential. Consider a group of test particles with the position $x$ and the velocity $\xi$ and their neighboring field particles located at $y \in \mathbb{R}^{3}-\{x\}$ with a velocity $\xi_{*}$. In this case, local net mass-momentum interactions between test particles at $x$ and neighboring field particles are measured by

$$
\mathcal{B}_{\varepsilon}^{\alpha \beta}(x, \xi, t):=m_{\alpha} F_{\alpha \varepsilon}(x, \xi)\left(\int_{\mathbb{R}^{6}} \nabla_{x} \omega(x-y) \cdot\left(\xi_{*}-\xi\right) m_{\beta} F_{\beta \varepsilon}\left(y, \xi_{*}\right) d \xi_{*} d y\right) .
$$

Then the generalized multi-dimensional Bony-type functional $\mathcal{B}(F(t))$ for $F=\left(F_{A}, F_{B}\right)$ and its time-production rate are defined as follows.

$$
\begin{aligned}
\mathcal{B}^{\alpha \beta}\left(F_{\varepsilon}(t)\right):= & \int_{\mathbb{R}^{6}} \mathcal{B}_{\varepsilon}^{\alpha \beta}(x, \xi, t) d \xi d x, \\
\mathcal{B}\left(F_{\varepsilon}(t)\right):= & \sum_{\alpha, \beta \in\{A, B\}} \mathcal{B}^{\alpha \beta}\left(F_{\varepsilon}(t)\right), \quad \mathcal{B}(F(t)):=\lim _{\varepsilon \rightarrow 0} \mathcal{B}\left(F_{\varepsilon}(t)\right), \\
\Lambda_{\mathcal{B}}^{\alpha \beta}\left(F_{\varepsilon}(t)\right):= & \sum_{\substack{1 \leq i, j \leq 3\\
}} \int_{\mathbb{R}^{12}}\left[\partial_{i} \partial_{j} \omega(x-y)\left(\xi_{*}-\xi\right)^{i}\left(\xi_{*}-\xi\right)^{j}\right] m_{\alpha} F_{\alpha \varepsilon}(x, \xi) m_{\beta} F_{\beta \varepsilon}\left(y, \xi_{*}\right) \\
& \times d y d x d \xi d \xi_{*}, \\
\Lambda_{\mathcal{B}}\left(F_{\varepsilon}(t)\right):= & \sum_{\alpha, \beta \in\{A, B\}} \Lambda_{\mathcal{B}}^{\alpha \beta}\left(F_{\varepsilon}(t)\right), \quad \Lambda_{\mathcal{B}}(F(t)):=\lim _{\varepsilon \rightarrow 0} \Lambda_{\mathcal{B}}\left(F_{\varepsilon}(t)\right) .
\end{aligned}
$$

Note that the functional $\mathcal{B}$ is well defined for each $t$ :

$$
|\mathcal{B}(t)| \leq 2\left\|\varphi^{\prime}\right\|_{L^{\infty}}\left\|\left(m_{A} F_{A}+m_{B} F_{B}\right)(t)\right\|_{L^{1}}^{\frac{3}{2}}\left\||\xi|^{2}\left(m_{A} F_{A}+m_{B} F_{B}\right)(t)\right\|_{L^{1}}^{\frac{1}{2}},
$$

which is bounded by the total mass and energy of initial datum $F_{A 0}$ and $F_{B 0}$ due to Proposition 3.1.

Lemma 7.1. Let $F_{\varepsilon}=\left(F_{A \varepsilon}, F_{B \varepsilon}\right)$ be mollified solutions to (4.1) with finite energy and mass. Then we have

$$
\begin{aligned}
\frac{d}{d t} \mathcal{B}^{\alpha \beta}\left(F_{\varepsilon}(t)\right) & \\
= & -\Lambda_{\mathcal{B}}^{\alpha \beta}\left(F_{\varepsilon}(t)\right) \\
+ & \int_{\mathbb{R}^{6}} \nabla_{x} \omega(x-y) \cdot\left[\left(\int m_{\alpha} F_{\alpha}(x, \xi) d \xi\right)\left(\int m_{\beta} \xi_{*} Q^{\beta \alpha}\left(F_{\beta \varepsilon}, F_{\alpha \varepsilon}\right)\left(y, \xi_{*}\right) d \xi_{*}\right)\right] d y d x \\
+ & \int_{\mathbb{R}^{12}} \nabla_{x} \omega(x-y) \cdot\left(\xi_{*}-\xi\right)\left[m_{\alpha} F_{\alpha}(x, \xi) m_{\beta}\left(P_{\varepsilon}^{\beta \beta}+P_{\varepsilon}^{\beta \alpha}\right)\left(y, \xi_{*}\right)\right. \\
& \left.+m_{\beta} F_{\beta}(x, \xi) m_{\alpha}\left(P_{\varepsilon}^{\alpha \alpha}+P_{\varepsilon}^{\alpha \beta}\right)\left(y, \xi_{*}\right)\right] d \xi_{*} d \xi d y d x .
\end{aligned}
$$

Proof. We first calculate the time derivative of integrand

$\partial_{t}\left(\left[\nabla_{x} \omega(x-y) \cdot\left(\xi_{*}-\xi\right)\right] F_{\alpha \varepsilon}(x, \xi) F_{\beta \varepsilon}\left(y, \xi_{*}\right)\right)$ 


$$
\begin{gathered}
=-\left[\nabla_{x} \omega(x-y) \cdot\left(\xi_{*}-\xi\right)\right] \operatorname{div}_{x}\left(\xi F_{\alpha \varepsilon}(x, \xi) F_{\beta \varepsilon}\left(y, \xi_{*}\right)\right) \\
-\left[\nabla_{x} \omega(x-y) \cdot\left(\xi_{*}-\xi\right)\right] \operatorname{div}_{y}\left(\xi_{*} F_{\alpha \varepsilon}(x, \xi) F_{\beta \varepsilon}\left(y, \xi_{*}\right)\right) \\
+\left[\nabla_{x} \omega(x-y) \cdot\left(\xi_{*}-\xi\right)\right] F_{\alpha \varepsilon}(x, \xi)\left(Q^{\beta \beta}\left(F_{\beta \varepsilon}, F_{\beta \varepsilon}\right)+Q^{\beta \alpha}\left(F_{\beta \varepsilon}, F_{\alpha \varepsilon}\right)+P_{\varepsilon}^{\beta \beta}+P_{\varepsilon}^{\beta \alpha}\right) \\
\times\left(y, \xi_{*}\right) \\
+\left[\nabla_{x} \omega(x-y) \cdot\left(\xi_{*}-\xi\right)\right] F_{\beta \varepsilon}\left(y, \xi_{*}\right)\left(Q^{\alpha \alpha}\left(F_{\alpha \varepsilon}, F_{\alpha \varepsilon}\right)+Q^{\alpha \beta}\left(F_{\alpha \varepsilon}, F_{\beta \varepsilon}\right)+P_{\varepsilon}^{\alpha \alpha}+P_{\varepsilon}^{\alpha \beta}\right) \\
\times(x, \xi) .
\end{gathered}
$$

We now integrate the above equation over $\mathbb{R}^{12}$ with respect to $\left(x, \xi, y, \xi_{*}\right)$ to get

$$
\begin{aligned}
\frac{d}{d t} \mathcal{B}^{\alpha \beta}\left(F_{\varepsilon}(t)\right) & \\
= & -\int_{\mathbb{R}^{12}}\left[\nabla_{x} \omega(x-y) \cdot\left(\xi_{*}-\xi\right)\right] \operatorname{div}_{x}\left(\xi m_{\alpha} F_{\alpha \varepsilon}(x, \xi) m_{\beta} F_{\beta \varepsilon}\left(y, \xi_{*}\right)\right) d \xi_{*} d \xi d y d x \\
& -\int_{\mathbb{R}^{12}}\left[\nabla_{x} \omega(x-y) \cdot\left(\xi_{*}-\xi\right)\right] \operatorname{div}_{y}\left(\xi_{*} m_{\alpha} F_{\alpha \varepsilon}(x, \xi) m_{\beta} F_{\beta \varepsilon}\left(y, \xi_{*}\right)\right) d \xi_{*} d \xi d y d x \\
& +\int_{\mathbb{R}^{12}}\left[\nabla_{x} \omega(x-y) \cdot\left(\xi_{*}-\xi\right)\right] m_{\alpha} F_{\alpha \varepsilon}(x, \xi) \\
& \times m_{\beta}\left(Q^{\beta \beta}\left(F_{\beta \varepsilon}, F_{\beta \varepsilon}\right)+Q^{\beta \alpha}\left(F_{\beta \varepsilon}, F_{\alpha \varepsilon}\right)+P_{\varepsilon}^{\beta \beta}+P_{\varepsilon}^{\beta \alpha}\right)\left(y, \xi_{*}\right) \times d \xi_{*} d \xi d y d x \\
& +\int_{\mathbb{R}^{12}}\left[\nabla_{x} \omega(x-y) \cdot\left(\xi_{*}-\xi\right)\right] m_{\beta} F_{\beta \varepsilon}\left(y, \xi_{*}\right) \\
& \times m_{\alpha}\left(Q^{\alpha \alpha}\left(F_{\alpha \varepsilon}, F_{\alpha \varepsilon}\right)+Q^{\alpha \beta}\left(F_{\alpha \varepsilon}, F_{\beta \varepsilon}\right)+P_{\varepsilon}^{\alpha \alpha}+P_{\varepsilon}^{\alpha \beta}\right)(x, \xi) \times d \xi_{*} d \xi d y d x \\
:= & \mathcal{I}_{1}+\mathcal{I}_{2}+\mathcal{I}_{3}+\mathcal{I}_{4} .
\end{aligned}
$$

Case $1\left(\mathcal{I}_{1}\right)$ and $\left(\mathcal{I}_{2}\right)$ : It is easy to check that

$$
\begin{aligned}
-\sum_{i, j} \partial_{i} \omega(x-y)\left(\xi_{*}-\xi\right)^{i} \partial_{x_{j}}\left(\xi^{j} m_{\alpha} F_{\alpha}(x, \xi) m_{\beta} F_{\beta}\left(y, \xi_{*}\right)\right) \\
=-\sum_{i, j} \partial_{x_{j}}\left(\partial_{i} \omega(x-y)\left(\xi_{*}-\xi\right)^{i} \xi^{j} m_{\alpha} F_{\alpha}(x, \xi) m_{\beta} F_{\beta}\left(y, \xi_{*}\right)\right) \\
+\sum_{i, j} \partial_{j} \partial_{i} \omega(x-y)\left(\xi_{*}-\xi\right)^{i} \xi^{j} m_{\alpha} F_{\alpha}(x, \xi) m_{\beta} F_{\beta}\left(y, \xi_{*}\right),
\end{aligned}
$$

and we use this relation to calculate

$$
\begin{aligned}
& \mathcal{I}_{1}=\int_{\mathbb{R}^{12}} \sum_{i, j} \partial_{j} \partial_{i} \omega(x-y)\left(\xi_{*}-\xi\right) \xi^{j} F_{\alpha}(x, \xi) m_{\beta} F_{\beta}\left(y, \xi_{*}\right) d \xi_{*} d \xi d y d x, \\
& \mathcal{I}_{2}=\int_{\mathbb{R}^{12}} \sum_{i, j}-\partial_{j} \partial_{i} \omega(x-y)\left(\xi_{*}-\xi\right) \xi_{*}^{j} F_{\alpha}(x, \xi) m_{\beta} F_{\beta}\left(y, \xi_{*}\right) d \xi_{*} d \xi d y d x,
\end{aligned}
$$

which gives $\mathcal{I}_{1}+\mathcal{I}_{2}=-\Lambda_{\mathcal{B}}^{\alpha \beta}\left(F_{\varepsilon}(t)\right)$.

Case $2\left(\mathcal{I}_{3}\right)$ and $\left(\mathcal{I}_{4}\right)$ : We can rewrite

$$
\begin{aligned}
& \int_{\mathbb{R}^{12}} \nabla_{x} \omega(x-y) \cdot\left(\xi_{*}-\xi\right) m_{\alpha} F_{\alpha}(x, \xi) m_{\beta}\left(Q^{\beta \beta}\left(F_{\beta \varepsilon}, F_{\beta \varepsilon}\right)+Q^{\beta \alpha}\left(F_{\beta \varepsilon}, F_{\alpha \varepsilon}\right)\right)\left(y, \xi_{*}\right) d \xi_{*} d \xi d y d x \\
&=\int_{\mathbb{R}^{6}} \nabla_{x} \omega(x-y) \cdot[\left(\int m_{\alpha} F_{\alpha}(x, \xi) d \xi\right)\left(\int m_{\beta} \xi_{*} Q^{\beta \beta}\left(F_{\beta \varepsilon}, F_{\beta \varepsilon}\right)\left(y, \xi_{*}\right) d \xi_{*}\right) \\
&+\left(\int m_{\alpha} F_{\alpha}(x, \xi) d \xi\right)\left(\int m_{\beta} \xi_{*} Q^{\beta \alpha}\left(F_{\beta \varepsilon}, F_{\alpha \varepsilon}\right)(y, \xi) d \xi_{*}\right)
\end{aligned}
$$




$$
\begin{aligned}
&-\left(\int m_{\alpha} \xi F_{\alpha}(x, \xi) d \xi\right)\left(\int m_{\beta} Q^{\beta \beta}\left(F_{\beta \varepsilon}, F_{\beta \varepsilon}\right)\left(y, \xi_{*}\right) d \xi_{*}\right) \\
&\left.-\left(\int m_{\alpha} \xi F_{\alpha}(x, \xi) d \xi\right)\left(\int m_{\beta} Q^{\beta \alpha}\left(F_{\beta \varepsilon}, F_{\alpha \varepsilon}\right)(y, \xi) d \xi_{*}\right)\right] d y d x \\
&=\int_{\mathbb{R}^{6}} \nabla_{x} \omega(x-y) \cdot\left(\int m_{\alpha} F_{\alpha}(x, \xi) d \xi\right)\left(\int m_{\beta} \xi_{*} Q^{\beta \alpha}\left(F_{\beta \varepsilon}, F_{\alpha \varepsilon}\right)\left(y, \xi_{*}\right) d \xi_{*}\right) d y d x
\end{aligned}
$$

where we use the local mass and momentum conservation from (i) and (ii) of Proposition 3.1 and $\mathcal{I}_{4}$ can be treated similarly to get the desired result.

Proposition 7.1. Let $F_{\varepsilon}=\left(F_{A \varepsilon}, F_{B \varepsilon}\right)$ be the mollified solution to (4.1) with a finite mass and energy. Then we have

$$
\frac{d}{d t} \mathcal{B}\left(F_{\varepsilon}(t)\right) \leq-\Lambda_{\mathcal{B}}\left(F_{\varepsilon}(t)\right)+C \int_{\mathbb{R}^{6}}(1+|\xi|) P(x, \xi, \varepsilon) d \xi d x, \quad t \geq 0
$$

Proof. From Lemma 7.1 we have

$$
\begin{aligned}
& \frac{d}{d t} \mathcal{B}\left(F_{\varepsilon}(t)\right) \\
& =-\Lambda_{\mathcal{B}}\left(F_{\varepsilon}(t)\right) \\
& \quad+\int_{\mathbb{R}^{6}} \nabla_{x} \omega(x-y) \cdot\left(\int\left(m_{A} F_{A}+m_{B} F_{B}\right)(x, \xi) d \xi\right) \\
& \quad \times\left(\int\left(m_{A} \xi_{*} Q^{A B}\left(F_{A \varepsilon}, F_{B \varepsilon}\right)+m_{B} \xi_{*} Q^{B A}\left(F_{B \varepsilon}, F_{A \varepsilon}\right)\right)\left(y, \xi_{*}\right) d \xi_{*}\right) d y d x \\
& \quad+\int_{\mathbb{R}^{12}} \nabla_{x} \omega(x-y) \cdot\left(\xi_{*}-\xi\right)\left(m_{A} F_{A}+m_{B} F_{B}\right)(x, \xi) \\
& \quad \quad \times\left(m_{A} P_{\varepsilon}^{A A}+m_{A} P_{\varepsilon}^{A B}+m_{B} P_{\varepsilon}^{B B}+m_{B} P_{\varepsilon}^{B A}\right)\left(y, \xi_{*}\right) d \xi_{*} d \xi d y d x \\
& \quad:=-\Lambda_{\mathcal{B}}\left(F_{\varepsilon}(t)\right)+\mathcal{J}_{1}+\mathcal{J}_{2} .
\end{aligned}
$$

Then it is easy to see that $\mathcal{J}_{1}=0$ by Proposition 3.1 ( $\mathrm{iii}$ ).

Case $\left(\mathcal{J}_{2}\right)$ : We rewrite $\left(\mathcal{J}_{2}\right)$ as

$$
\begin{aligned}
\mathcal{J}_{2}=\int_{\mathbb{R}^{6}} \nabla_{x} \omega(x & -y) \cdot\left(\int\left(m_{A} F_{A}+m_{B} F_{B}\right)(x, \xi) d \xi\right) \\
& \times\left(\int \xi_{*}\left(m_{A} P_{\varepsilon}^{A A}+m_{A} P_{\varepsilon}^{A B}+m_{B} P_{\varepsilon}^{B B}+m_{B} P_{\varepsilon}^{B A}\right)\left(y, \xi_{*}\right) d \xi_{*}\right) d y d x \\
+\int_{\mathbb{R}^{6}} \nabla_{x} \omega(x & -y) \cdot\left(\int \xi\left(m_{A} F_{A}+m_{B} F_{B}\right)(x, \xi) d \xi\right) \\
& \times\left(\int\left(m_{A} P_{\varepsilon}^{A A}+m_{A} P_{\varepsilon}^{A B}+m_{B} P_{\varepsilon}^{B B}+m_{B} P_{\varepsilon}^{B A}\right)\left(y, \xi_{*}\right) d \xi_{*}\right) d y d x,
\end{aligned}
$$

and then we can show that

$$
\begin{aligned}
\left|\mathcal{J}_{2}\right| & \leq\left(m_{A}+m_{B}\right)\left\|\varphi^{\prime}\right\|_{L^{\infty}}\left\|\left(m_{A} F_{A}+m_{B} F_{B}\right)(t)\right\|_{L^{1}} \int_{\mathbb{R}^{6}}|\xi| P(x, \xi, \varepsilon) d \xi d x \\
& +\left(m_{A}+m_{B}\right)\left\|\varphi^{\prime}\right\|_{L^{\infty}}\left\||\xi|\left(m_{A} F_{A}+m_{B} F_{B}\right)(t)\right\|_{L^{1}} \int_{\mathbb{R}^{6}} P(x, \xi, \varepsilon) d \xi d x \\
& \leq\left(m_{A}+m_{B}\right)\left\|\varphi^{\prime}\right\|_{L^{\infty}}\left\|\left(m_{A} F_{A}+m_{B} F_{B}\right)(t)\right\|_{L^{1}}^{\frac{1}{2}} \\
& \times\left[\left\|\left(m_{A} F_{A}+m_{B} F_{B}\right)(t)\right\|_{L^{1}}^{\frac{1}{2}}+\left\|\left.\xi\right|^{2}\left(m_{A} F_{A}+m_{B} F_{B}\right)(t)\right\|_{L^{1}}^{\frac{1}{2}}\right] \int_{\mathbb{R}^{6}}(1+|\xi|) P(x, \xi, \varepsilon) d \xi d x .
\end{aligned}
$$

Finally, we return to the proof of Theorem 2.2. 
Proof of Theorem 2.2. From Proposition 5.1 we have

$$
\frac{d}{d t} \mathcal{B}\left(F_{\varepsilon}(t)\right) \leq-\Lambda_{\mathcal{B}}\left(F_{\varepsilon}(t)\right)+C \int_{\mathbb{R}^{6}}(1+|\xi|) P(x, \xi, \varepsilon) d \xi d x,
$$

where $C$ is independent of time $t$ and the mollification parameter $\varepsilon$ depends only on initial mass and energy. We now integrate (7.2) from $s=0$ to $s=t$ to get

$$
\mathcal{B}\left(F_{\varepsilon}(t)\right)+\int_{0}^{t} \Lambda_{\mathcal{B}}\left(F_{\varepsilon}(s)\right) d s \leq \mathcal{B}\left(F_{\varepsilon}(0)\right)+C \int_{0}^{t} \int_{\mathbb{R}^{6}}(1+|\xi|) P(x, \xi, \varepsilon) d \xi d x d s .
$$

We pass to the limit $\varepsilon \rightarrow 0$ to get

$$
\mathcal{B}(F(t))+\int_{0}^{t} \Lambda_{\mathcal{B}}(F(s)) d s \leq \mathcal{B}(F(0)) .
$$

This yields

$$
\begin{aligned}
\int_{0}^{\infty} & \int_{\mathbb{R}^{12}} \sum_{i, j} \partial_{j} \partial_{i} \omega(x-y)\left(\xi_{*}-\xi\right)^{i}\left(\xi_{*}-\xi\right)^{j} \\
& \quad \times\left(m_{A} F_{A}+m_{B} F_{B}\right)(x, \xi)\left(m_{A} F_{A}+m_{B} F_{B}\right)\left(y, \xi_{*}\right) d \xi_{*} d \xi d y d x \\
\leq & |\mathcal{B}(F(0))|+|\mathcal{B}(F(t))| \\
& \leq 4\left\|\varphi^{\prime}\right\|_{L^{\infty}}\left\|\left(m_{A} F_{A 0}+m_{B} F_{B 0}\right)\right\|_{L^{1}}^{3 / 2}\left\||\xi|^{2}\left(m_{A} F_{A 0}+m_{B} F_{B 0}\right)\right\|_{L^{1}}^{\frac{1}{2}}<\infty .
\end{aligned}
$$

This completes the proof.

REMARK 7.1. In [20], the special choice of a weight function $\omega=|x-y|$ was employed in the construction of the Bony functional.

\section{REFERENCES}

[1] Alonso, R.: Existence of global solutions to the Cauchy problem for the inelastic Boltzmann equation with near-vacuum data. To appear in Indiana Univ. Math. J.

[2] Andries, P., Aoki, K. and Perthame, B.: A consistent BGK-type model for gas mixtures. J. Stat. Phys. 106, 993-1018 (2002). MR1889599(2002k:82062)

[3] Aoki, K., Bardos, C. and Takata, S.: Knudsen layer for gas mixtures. J. Stat. Phys. 112, 629-655 (2003). MR 1997264 (2004f:82065)

[4] Bellomo, N. and Toscani, G.: On the Cauchy problem for the nonlinear Boltzmann equation: global existence, uniqueness and asymptotic behavior. J. Math. Phys. 26, 334-338 (1985). MR776503 (86h:82038)

[5] Benedetto, D. and Pulvirenti, M.: On the one-dimensional Boltzmann equation for granular flow. Math. Model. Numer. Anal. 35, 899-905 (2001). MR1866273 (2002h:82066)

[6] Bobylev, A. V. and Cercignani, C.: Self-similar asymptotics for the Boltzmann equation with inelastic and elastic interactions. J. Stat. Phys. 110, 333-375 (2003). MR1966332 (2004g:82110)

[7] Bobylev, A. V., Cercignani, C. and Toscani, G.: Proof of an asymptotic property of self-similar solutions of the Boltzmann equation for granular materials. J. Stat. Phys. 111, 403-417 (2003). MR 1964277(2004d:82032)

[8] Bobylev, A. V., Carrillo, J. A. and Gamba, I. M.: On some properties of kinetic and hydrodynamic equations for inelastic interactions. J. Stat. Phys. 98, 743-773 (2000). MR1749231(2001c:82063)

[9] Bobylev, A. V., Gamba, I. M. and Panferov, V. A.: Moment inequalities and high-energy tails for Boltzmann equations with inelastic interactions. J. Stat. Phys. 116, 1651-1682 (2004). MR2096050 (2005g:82111)

[10] Bony, J. M.: Solutions globales bornées pour les modèles discrete de l'équation de Boltzmann en dimension 1 d'espace. Acte Journees E. D. P. St. Jean de Monts, $n^{o}$ XVI (1987).

[11] Cercignani, C., Illner, R. and Stoica, C.: On diffusive equilibria in generalized kinetic theory. J. Stat. Phys. 105, 337-352 (2001). MR.1861207(2002j:82093)

[12] Chapman, S. and Cowling, T. G.: The mathematical theory of non-uniform gases. Cambridge University Press, London (1952). 
[13] Chae, M. and Ha, S.-Y.: Stability estimates of the Boltzmann equation with quantum effects. Contin. Mech. Thermodyn. 17, 511-524 (2006). MR2240606 (2007j:82095)

[14] Gamba, I. M., Panferov, V. and Villani, C.: On the Boltzmann equation for diffusively excited granular media. Comm. Math. Phys. 246, 503-541 (2004). MR2053942 (2005b:82076)

[15] Garzo, V., Santos, A. and Brey, J. J.: A kinetic model for a multi-component gas. Phys. Fluids A 1, 380-383 (1989).

[16] Jin, S. and Slemrod, M.: Regularization of the Burnett equations for rapid granular flows via relaxation. Physica D 150, 207-218 (2001). MR.1820735 (2001k:76094)

[17] Ha, S.-Y.: $L^{1}$ stability estimate for a one-dimensional Boltzmann equation with inelastic collisions. J. Differential Equations 190, 621-642 (2003). MR:1971148(2004h:35035)

[18] Ha, S.-Y.: Nonlinear functionals of the Boltzmann equation and uniform stability estimates. J. Differential Equations 215, 178-205 (2005). MR2146347 (2006b:35033)

[19] Ha, S.-Y. and Yun, S.-B.: Uniform $L^{1}$-stability estimate of the Boltzmann equation near a local Maxwellian. Physica D 220, 79-97 (2006). MR2252152 (2007f:82082)

[20] Ha, S.-Y., and Noh, S. E.: New a priori estimate for the Boltzmann-Enskog equation. Nonlinearity 19, 1219-1232 (2006). MR.2229996 (2009c:35363)

[21] Hamel, B.: Kinetic model for binary gas mixtures. Phys. Fluids 8, 418-425 (1965).

[22] Illner, R. and Shinbrot, M.: Global existence for a rare gas in an infinite vacuum. Comm. Math. Phys. 95, 217-226 (1984). MR760333 (86a:82019)

[23] Kaniel, S. and Shinbrot, M.: The Boltzmann equation 1: Uniqueness and local existence. Comm. Math. Phys. 58, 65-84 (1978). MR0475532 (57:15133)

[24] Polewczak, J.: Classical solution of the nonlinear Boltzmann equation in all $R^{3}$ : asymptotic behavior of solutions. J. Stat. Phys. 50, 611-632 (1988). MR.939503 (89h:82021)

[25] Sone, Y.: Kinetic theory and fluid dynamics. Birkhauser, Boston 2002. MR.1919070 (2003h:76113)

[26] Sone, Y.: Molecular gas dynamics. Birkhauser, Boston 2006. MR 2274674 (2007m:82081)

[27] Villani, C.: Mathematics of granular materials. J. Stat. Phys. 124, 781-822 (2006). MR2264625 $(2007 \mathrm{j}: 82116)$

[28] Villani, C.: A review of mathematical topics in collisional kinetic theory. Handbook of Fluid Mechanics, S. Friedlander and D. Serre, Eds. (2001). MR.1942465 (2003k:82087)

[29] Yasuda, S., Takata, S. and Aoki, K.: Evaporation and condensation of a binary mixture of vapors on a plane condensed phase: Numerical analysis of the linearized Boltzmann equation. Phys. Fluids 17, 047105 (2005).

[30] Yasuda, S., Takata S. and Aoki, K.: Numerical analysis of the shear flow of a binary mixture of hard-sphere gases over a plane wall. Phys. Fluids 16, 1989-2003 (2004). 